\title{
Pd-Catalyzed Amination for the Synthesis of Macropolycycles Comprising Cyclen, Cyclam and Naphthalene Moieties
}

\author{
A. D. Averin, ${ }_{1}{ }^{@}$ A. V. Shukhaev, ${ }^{a}$ A. I. $\operatorname{Vovk}{ }^{\text {b }}$ V. P. Kukhar, ${ }^{b}$ F. Denat, ${ }^{c}$ R. Guilard ${ }^{c}$ \\ and I. P. Beletskayab
}

Dedicated to Corresponding member of Russian Academy of Sciences Prof. Oscar Koifman on the occasion of his $70^{\text {th }}$ Anniversary

\begin{abstract}
a'Lomonosov Moscow State University, Department of Chemistry, 119991 Moscow, Russia
'Institute of Bioorganic Chemistry and Petrol Chemistry of Ukraine NAS, 02660 Kiev, Ukraine

'Institut de Chimie Moléculaire de l'Université de Bourgogne (ICMUB), 21078 Dijon Cedex, France

${ }^{\circledR}$ Corresponding authorE-mail: averin@org.chem.msu.ru
\end{abstract}

\begin{abstract}
Pd-catalyzed amination reactions were employed for the synthesis of macrobicyclic compounds possessing cyclen or cyclam moieties, naphthylmethyl spacers and polyamine linkers. The results of the macrocyclization reactions involving 1,7-bis(4-bromonaphthyl-1-methyl)cyclen and 1,8-bis(4-bromonaphthyl-1-methyl)cyclam were shown to be dependent on the nature of starting tetraazamacrocycles and polyamines, the better yields being observed in the case of cyclen derivatives. Valuable macrotricyclic compounds were obtained as the second products in the reactions of 1,7-bis(4bromonaphthyl-1-methyl)cyclen with the majority of polyamines.
\end{abstract}

Keywords: Pd-Catalized amination, macropolycycles, cyclen, cyclam.

\section{Рd-Катализируемое аминирование в синтезе макрополициклических соединений с фрагментами циклена, циклама и нафталина}

\author{
А. А. Аверин, ${ }^{a}{ }^{@}$ А. В. Шухаев, ${ }^{a}$ А. И. Вовк, ${ }^{\text {b }}$ В. П. Кухар, ${ }^{\text {b }}$ Ф. Аена, ${ }^{c}$ Р. Гиляр, ${ }^{c}$ \\ И. П. Белецкая
}

Посвящается Член-корреспонденту РАН профессору Оскару Иосифовичу Койфману по случаю его 70-летнего юбилея

\footnotetext{
а Московский государственный университет им. М.В. Ломоносова, Химический факультет, 119991 Москва, Россия ${ }^{\mathrm{b}}$ Институт биоорганической химии и нефтехимии НАН Украинь, 02660 Киев, Украина

' Институт молекулярной химии Университета Бургундии, 21078 Дижон, Франщия

${ }^{\circledR}$ E-mail: averin@org.chem.msu.ru
}

\begin{abstract}
Реакиии Рd-катализируемого аминирования использованы для синтеза макробициклических соединений, содержащих фрагменты цииклена или ичклама, нафтилметильные спейсеры и полиаминовые линкеры. Показано, что результат реакции макрочиклизации с участием 1,7-бис(4-бромнафтил-1-метил)цииклена и 1,8-бис(4-бромнафтил-1-метил)циклама зависят от природы исходных тетраазамакроциклов и полиаминов, лучшие выходы наблюдались для производных ииклена. Ценные макротрициклические соединения получены в качестве вторых продуктов реакций 1,7-бис(4-бромнафтил-1-метил)циклена с большинством полиаминов.
\end{abstract}

Ключевые слова: Pd-Катализируемое аминирование, макрополициклические соединения, циклен, циклам. 


\section{Introduction}

The first macrocycles incorporating naphthalene moieties were described in $1930 \mathrm{~s},{ }^{[1]}$ and since that time dozens of works appeared in which various macrocyclic compounds bearing this endocyclic fragment were described. Macrocycles containing 2,3-disubstituted naphthalene fragment have been investigated for alkali metals, ammonium and alkylammonium salts coordination, ${ }^{[2-7]}$ as well as macrocycles possessing two naphthalene and two polyoxa fragments. ${ }^{[8,9]}$ Macrocycles with polyoxa chains were obtained on the basis of 1,51,7- and 1,8-disubstituted naphthalene and their complexing properties towards alkali metals were studied. ${ }^{[5-10]}$ Several works deal with polyoxamacrocycles organized around 2,2'and 3,3'-disubstituted 1,1'-binaphthalenes, ${ }^{[6,7]}$ among these compounds there are sophisticated macrotricyclic cryptands bearing two 1,10-diaza-18-crown-6 fragments. ${ }^{[1]}$ Spherands with 2,2'-dioxy-1,1'-binaphthalenes and 2,7-disubstituted 1,8-dihydroxynaphtahlene moieties constitute another class of macrocycles, ${ }^{[10,12]}$ the latter compound was tested in the coordination studies with $\mathrm{Mg}(\mathrm{II}), \mathrm{Fe}(\mathrm{II}), \mathrm{Co}(\mathrm{II}), \mathrm{Ni}(\mathrm{II})$ and $\mathrm{Zn}$ (II) cations. Various nitrogen-containing macrocycles with naphthalene moieties were reported, among them are cyclic Schiff bases, ${ }^{[13]}$ diamides and diimides, ${ }^{[14,15]}$ lactams; ${ }^{[16]}$ naphthalene fragments were also condensed with tetraazamacrocycles. ${ }^{[17]}$ Naphthalene fragments were combined with calixarenes, ${ }^{[18]}$ catenanes, ${ }^{[19]}$ and porphyrins. ${ }^{[20]}$ Besides coordination with alkali cations, naphthalene-containing macrocycles were used for the formation of anion receptors and molecular rotors. ${ }^{[21,22]}$

\section{Experimental}

NMR spectra were registered using Bruker Avance 400 spectrometer, MALDI-TOF spectra were obtained with Bruker Ultraflex spectrometer using 1,8,9-trihydroxyanthracene as matrix and PEGs as internal standards. 1-Bromo-4-methylnaphthalene, di- and polyamines 7a-j, 2-(dicyclohexylphosphino)-2'(dimethylamino)-1,1'-biphenyl (DavePhos ligand), sodium tertbutoxide were purchased from Aldrich and Acros and used without further purification. Cis-glyoxal-cyclen $\mathbf{1}$ and bis-formaldehydecyclam 2 were provided by CheMatech Co. 1-Bromo-4(bromomethyl)naphthalene was synthesized in $90 \%$ yield from 1-bromo-4-methylnaphthalene according to a standard procedure using bromination with $\mathrm{Br}_{2}$ in $\mathrm{CCl}_{4}$. $\mathrm{Pd}(\mathrm{dba})_{2}$ was synthesized according to a known method. ${ }^{[23]}$ Dioxane was distilled over $\mathrm{NaOH}$ followed by the distillation over sodium under argon, acetonitrile was distilled over $\mathrm{CaH}_{2}$, dichloromethane and methanol were used freshly distilled.

2a,6a-Bis[(4-bromo-1-naphthyl)methyl]decahydro-4a,8adioxa-2a,6a-diazoniacyclopenta-[fg]acenaphtene dibromide (3). A flask equipped with a reflux condenser and magnetic stirrer was charged with cis-glyoxal-cyclen 1 (1.00 g, $5.1 \mathrm{mmol})$, 1-bromo4-(bromomethyl)naphthalene $(3.06 \mathrm{~g}, 10.2 \mathrm{mmol})$ and $17 \mathrm{~mL}$ of acetonitrile. The mixture was heated at $50-60{ }^{\circ} \mathrm{C}$ for $80 \mathrm{~h}$, the white precipitate formed was filtered off, washed with cold acetonitrile $(2 \times 15 \mathrm{~mL})$ and dried in vacuo at $100{ }^{\circ} \mathrm{C}$. Yield $3.58 \mathrm{~g}(88 \%) .{ }^{1} \mathrm{H}$ NMR (DMSO- $\left.d_{6}, 298 \mathrm{~K}\right) \delta_{\mathrm{H}}$ ppm: 2.91-2.99 $(2 \mathrm{H}, \mathrm{m}), 3.57(2 \mathrm{H}, \mathrm{t}$, $J=11.7 \mathrm{~Hz}), 3.64-3.83(6 \mathrm{H}, \mathrm{m}), 3.93\left(2 \mathrm{H}, \mathrm{td}, J=11.1 \mathrm{~Hz},{ }^{3} J=4.5\right.$ $\mathrm{Hz}), 5.57(2 \mathrm{H}, \mathrm{s}), 5.62(2 \mathrm{H}, \mathrm{s}), 7.81-7.86(2 \mathrm{H}, \mathrm{m}), 7.86-7.91(2 \mathrm{H}$, $\mathrm{m}), 7.97\left(2 \mathrm{H}, \mathrm{d},{ }^{3} J=7.8 \mathrm{~Hz}\right), 8.11\left(2 \mathrm{H}, \mathrm{d},{ }^{3} J=7.7 \mathrm{~Hz}\right), 8.32(2 \mathrm{H}$, d, $\left.{ }^{3} J=8.2 \mathrm{~Hz}\right), 8.69\left(2 \mathrm{H}, \mathrm{d},{ }^{3} J=8.1 \mathrm{~Hz}\right)(6$ protons in $3.30-3.40$ ppm region are overlapped by the signal of $\mathrm{H}_{2} \mathrm{O}$ of the solvent). ${ }^{13} \mathrm{C}$
NMR (DMSO- $\left.d_{6}, 298 \mathrm{~K}\right) \delta_{\mathrm{c}} \mathrm{ppm:} 42.5$ (2C), 46.1 (2C), 55.9 (2C), $56.3(2 \mathrm{C}), 59.6(2 \mathrm{C}), 76.0(2 \mathrm{C}), 123.9(2 \mathrm{C}), 124.7(2 \mathrm{C}), 126.1(2 \mathrm{C})$, 127.8 (2C), 128.5 (2C), 128.8 (2C) 129.9 (2C), 131.7 (2C), 134.0 (2C), $134.3(2 \mathrm{C})$.

$N^{1}, N^{7}$-di((4-bromonaphth-1-yl)methyl)cyclen (5). A flask equipped with a reflux condenser and magnetic stirrer was charged with disalt 3 (3.58 g, $4.45 \mathrm{mmol}), \mathrm{KOH}(8.1 \mathrm{~g}, 0.145 \mathrm{~mol})$ and $50 \mathrm{~mL}$ of water. The mixture was stirred at $80-90{ }^{\circ} \mathrm{C}$ for $72 \mathrm{~h}$, cooled down to ambient temperature, extracted with $\mathrm{CH}_{2} \mathrm{Cl}_{2}(3 \times 50$ $\mathrm{mL}$ ). The organic phase was dried over $\mathrm{Na}_{2} \mathrm{SO}_{4}$, the solvent was evaporated in vacuo, and the product was obtained as a beige crystalline powder. Yield $2.68 \mathrm{~g}(99 \%)$, m.p. $133-135^{\circ} \mathrm{C}$. (MALDITOF) found: 609.1274. $\mathrm{C}_{30} \mathrm{H}_{35} \mathrm{Br}_{2} \mathrm{O}_{4}$ requires $609.1228[\mathrm{M}+\mathrm{H}]^{+}$ $\mathrm{UV}\left(\mathrm{CH}_{2} \mathrm{Cl}_{2}\right) \lambda_{\max } \mathrm{nm}(\varepsilon): 292$ (14000). ${ }^{1} \mathrm{H} \mathrm{NMR}\left(\mathrm{CDCl}_{3}, 298 \mathrm{~K}\right)$ $\delta_{\mathrm{H}}$ ppm: $2.59-2.67(16 \mathrm{H}, \mathrm{m}), 3.96(4 \mathrm{H}, \mathrm{s}), 7.22\left(2 \mathrm{H}, \mathrm{d},{ }^{3} J=7.6 \mathrm{~Hz}\right)$, $7.43\left(2 \mathrm{H}, \mathrm{t},{ }^{3} \mathrm{~J}_{\text {obs }}=7.6 \mathrm{~Hz}\right), 7.55\left(2 \mathrm{H}, \mathrm{t},{ }^{3} J_{\text {obs }}=7.7 \mathrm{~Hz}\right), 7.61(2 \mathrm{H}$, $\left.\mathrm{d},{ }^{3} J=7.6 \mathrm{~Hz}\right), 8.10\left(2 \mathrm{H}, \mathrm{d},{ }^{3} J=8.5 \mathrm{~Hz}\right), 8.28\left(2 \mathrm{H}, \mathrm{d},{ }^{3} J=8.4 \mathrm{~Hz}\right)$ (two NH protons were not assigned). ${ }^{13} \mathrm{C}$ NMR $\left(\mathrm{CDCl}_{3}, 298 \mathrm{~K}\right) \delta$ ppm: 45.7 (4C), 52.3 (4C), 58.1 (2C), 122.5 (2C), $123.8(2 \mathrm{C}), 126.8$ (2C), 126.9 (2C), 127.6 (2C), 127.9 (2C), 129.4 (2C), 132.0 (2C), 133.4 (2C) 134.5 (2C).

1,8-Bis [(4-bromo-1-naphthyl)methyl]-4,11-diaza-1,8diazoniatricyclo[9.3.1.14,8]hexadecane dibromide (4). A flask equipped with a magnetic stirrer was charged with bis-formaldehydecyclam 2 (2.5 g, $11.2 \mathrm{mmol})$, 1-bromo-4-(bromomethyl)naphthalene (6.9 g, $23 \mathrm{mmol})$ and $75 \mathrm{~mL}$ of acetonitrile. The reaction mixture was stirred at room temperature for $24 \mathrm{~h}$, the white precipitate formed was filtered off, washed with cold acetonitrile $(3 \times 50 \mathrm{~mL})$ and dried in vacuo at $100{ }^{\circ} \mathrm{C}$. Yield $7.05 \mathrm{~g}(76 \%)$. The compound is almost insoluble in common solvents like $\mathrm{D}_{2} \mathrm{O}, \mathrm{CD}_{3} \mathrm{OD}$ and DMSO- $d_{6}$, thus NMR spectra were not recorded and the compound was used directly in the second step.

$N^{l}, N^{\top}$-di((4-bromonaphth-1yl)methyl)cyclam (6). A flask equipped with a reflux condenser and magnetic stirrer was charged with disalt 4 (7.05 g, $8.56 \mathrm{mmol}), \mathrm{NaOH}(14 \mathrm{~g}, 0.35 \mathrm{mmol})$ and 100 $\mathrm{mL}$ of water. The mixture was stirred at $90^{\circ} \mathrm{C}$ for $48 \mathrm{~h}$, cooled down to ambient temperature, extracted with $\mathrm{CH}_{2} \mathrm{Cl}_{2}(3 \times 50 \mathrm{~mL})$. The organic phase was dried over $\mathrm{Na}_{2} \mathrm{SO}_{4}$, the solvent was evaporated in vacuo, and the product was obtained as a yellowish crystalline powder. Yield $3.32 \mathrm{~g}(61 \%)$, m.p. 137-139 ${ }^{\circ} \mathrm{C}$. (MALDI-TOF) found: 637.1517. $\mathrm{C}_{32} \mathrm{H}_{39} \mathrm{Br}_{2} \mathrm{~N}_{4}$ requires 637.1541 [M+H] $]^{+}$. UV $\left(\mathrm{CH}_{2} \mathrm{Cl}_{2}\right) \lambda_{\max } \mathrm{nm}(\varepsilon): 292 \mathrm{~nm}(13000) .{ }^{1} \mathrm{H} \mathrm{NMR}\left(\mathrm{CDCl}_{3}, 298 \mathrm{~K}\right) \delta_{\mathrm{H}}$ ppm: 1.77 (4H, quintet, $\left.{ }^{3} \mathrm{~J}=5.4 \mathrm{~Hz}\right), 2.53\left(4 \mathrm{H}, \mathrm{t},{ }^{3} \mathrm{~J}=5.4 \mathrm{~Hz}\right), 2.57$ $\left(4 \mathrm{H}, \mathrm{t},{ }^{3} \mathrm{~J}=5.7 \mathrm{~Hz}\right), 2.61-2.66(4 \mathrm{H}, \mathrm{m}), 2.68-2.72(4 \mathrm{H}, \mathrm{m}), 3.91(4 \mathrm{H}$, s), $7.27\left(2 \mathrm{H}, \mathrm{d},{ }^{3} J=7.8 \mathrm{~Hz}\right), 7.53-7.61(6 \mathrm{H}, \mathrm{m}), 8.21-8.25(2 \mathrm{H}, \mathrm{m})$, 8.36-8.40 (2H, m) (two $\mathrm{NH}$ protons were not assigned). ${ }^{13} \mathrm{C}$ NMR $\left(\mathrm{CDCl}_{3}, 298 \mathrm{~K}\right) \delta_{\mathrm{c}} \mathrm{ppm}: 26.3(2 \mathrm{C}), 47.8(2 \mathrm{C}), 48.5(2 \mathrm{C}), 52.7(2 \mathrm{C})$, $53.7(2 \mathrm{C}), 57.0(2 \mathrm{C}), 122.4(2 \mathrm{C}), 124.6(2 \mathrm{C}), 126.6(2 \mathrm{C}), 127.0$ (2C), 127.5 (2C), 127.8 (2C), 129.2 (2C), 132.0 (2C), 133.4 (2C), $134.7(2 \mathrm{C})$.

Typical procedure for the synthesis of macrobicycles $8,9$. A two-neck flask equipped with a reflux condenser and magnetic stirrer, flushed with dry argon, was charged with compound $\mathbf{5}$ or 6 (0.5 mmol), Pd(dba) (44 mg, $16 \mathrm{~mol} \%$ ), DavePhos ligand (32 $\mathrm{mg}, 16 \mathrm{~mol} \%)$, absolute dioxane $(25 \mathrm{~mL})$, the mixture was stirred for 2-3 $\mathrm{min}$, then appropriate polyamine $7(0.5 \mathrm{mmol})$ was added followed by $t$-BuONa (144 mg, $1.5 \mathrm{mmol})$. The reaction mixture was stirred at reflux for $24 \mathrm{~h}$, cooled down to ambient temperature, the solvent was filtered and the residue washed with $\mathrm{CH}_{2} \mathrm{Cl}_{2}(3 \times 5$ $\mathrm{mL}$ ), combined organic fractions were evaporated in vacuo and the oily residue chromatographed on silica gel using a sequence of eluents: $\mathrm{CH}_{2} \mathrm{Cl}_{2}, \mathrm{CH}_{2} \mathrm{Cl}_{2} / \mathrm{MeOH}$ (50:1-3:1), $\mathrm{CH}_{2} \mathrm{Cl}_{2} / \mathrm{MeOH} / \mathrm{NH}_{3}$ aq (100:20:1-10:4:1).

Macrobicycle 8a. Obtained from compound 5 (304 mg, 0.5 $\mathrm{mmol})$ and diamine $7 \mathbf{a}(37 \mathrm{mg}, 0.5 \mathrm{mmol})$. Eluent $\mathrm{CH}_{2} \mathrm{Cl}_{2} / \mathrm{MeOH}$ 3:1. Yield $54 \mathrm{mg}(20 \%)$, light-beige crystalline powder, m.p. 183-185 ${ }^{\circ} \mathrm{C}$. (MALDI-TOF) found: 523.3506. $\mathrm{C}_{33} \mathrm{H}_{43} \mathrm{~N}_{6}$ requires $523.3549[\mathrm{M}+\mathrm{H}]^{+}$. UV $\left(\mathrm{CH}_{2} \mathrm{Cl}_{2}\right) \lambda_{\max } \mathrm{nm}(\varepsilon): 340(8300) .{ }^{1} \mathrm{H}$ NMR 
$\left(\mathrm{CDCl}_{3}, 298 \mathrm{~K}\right) \delta_{\mathrm{H}} \mathrm{ppm}: 2.04\left(2 \mathrm{H}\right.$, quintet, $\left.{ }^{3} J=6.8 \mathrm{~Hz}\right), 2.35-2.44$ (4H, m), 2.68-2.76 (4H, m), 2.76-2.85 (4H, m), 2.90-2.97 (4H, m), $3.59\left(4 \mathrm{H}, \mathrm{t},{ }^{3} \mathrm{~J}=6.8 \mathrm{~Hz}\right), 3.98(4 \mathrm{H}, \mathrm{s}), 4.83(2 \mathrm{H}, \mathrm{br} . \mathrm{s}), 6.49\left(2 \mathrm{H}, \mathrm{d},{ }^{3} \mathrm{~J}\right.$ $=7.8 \mathrm{~Hz}), 6.97\left(2 \mathrm{H}, \mathrm{d},{ }^{3} J=7.8 \mathrm{~Hz}\right), 7.42\left(2 \mathrm{H}, \mathrm{t},{ }^{3} J_{\text {obs }}=7.6 \mathrm{~Hz}\right), 7.50$ $\left(2 \mathrm{H}, \mathrm{t},{ }^{3} J_{o b s}=7.6 \mathrm{~Hz}\right), 7.83\left(2 \mathrm{H}, \mathrm{d},{ }^{3} J=8.4 \mathrm{~Hz}\right), 8.04\left(2 \mathrm{H}, \mathrm{d}^{3} J=8.4\right.$ $\mathrm{Hz})$ (two NH protons were not assigned). ${ }^{13} \mathrm{C} \mathrm{NMR}\left(\mathrm{CDCl}_{3}, 298 \mathrm{~K}\right)$ $\delta_{\mathrm{c}}$ ppm: 27.2 (1C), 41.1 (2C), 47.1 (4C), 51.8 (4C), 57.7 (2C), 105.0 (2C), 120.5 (2C), 123.4 (2C), 123.5 (2C), 123.8 (2C), $124.4(2 \mathrm{C})$, 125.9 (2C), 128.2 (2C), 133.1 (2C), 142.1 (2C).

Cyclodimer 10a. Obtained as the second product in the synthesis of macrobicycle 8a. Eluent $\mathrm{CH}_{2} \mathrm{Cl}_{2} / \mathrm{MeOH} / \mathrm{NH}_{3}$ aq 100:20:1. Yield $44 \mathrm{mg}(17 \%)$, beige glassy compound. (MALDITOF) found: 1045.56. $\mathrm{C}_{66} \mathrm{H}_{85} \mathrm{~N}_{12}$ requires $1045.70[\mathrm{M}+\mathrm{H}]^{+} .{ }^{1} \mathrm{H} \mathrm{NMR}$ $\left(\mathrm{CDCl}_{3}, 298 \mathrm{~K}\right) \delta_{\mathrm{H}}$ ppm: $1.96\left(4 \mathrm{H}\right.$, quintet, $\left.{ }^{3} J=6.7 \mathrm{~Hz}\right), 2.51-2.70$ $(32 \mathrm{H}, \mathrm{m}), 3.51(8 \mathrm{H}$, br.s $), 3.88(8 \mathrm{H}, \mathrm{s}), 4.64(4 \mathrm{H}, \mathrm{br} . \mathrm{s}), 6.44(4 \mathrm{H}, \mathrm{d}$, $\left.{ }^{3} J=8.0 \mathrm{~Hz}\right), 6.97\left(4 \mathrm{H}, \mathrm{d},{ }^{3} J=8.0 \mathrm{~Hz}\right), 7.35\left(4 \mathrm{H}, \mathrm{t},{ }^{3} J_{o b s}=7.9 \mathrm{~Hz}\right)$, $7.47\left(4 \mathrm{H}, \mathrm{t},{ }^{3} J_{o b s}=7.6 \mathrm{~Hz}\right), 7.77\left(4 \mathrm{H}, \mathrm{d},{ }^{3} J=8.7 \mathrm{~Hz}\right), 8.04\left(4 \mathrm{H}, \mathrm{d},{ }^{3} \mathrm{~J}\right.$ $=8.7 \mathrm{~Hz}$ ) (four $\mathrm{NH}$ protons were not assigned).

Macrobicycle 8b. Obtained from compound 5 (304 mg, 0.5 $\mathrm{mmol}$ ) and diamine $7 \mathbf{b}$ (44 mg, $0.5 \mathrm{mmol})$. Eluent $\mathrm{CH}_{2} \mathrm{Cl}_{2} / \mathrm{MeOH}$ 3:1. Yield $19 \mathrm{mg}(7 \%)$, beige crystalline powder, m.p. $163-165^{\circ} \mathrm{C}$. (MALDI-TOF) found: 537.3741. $\mathrm{C}_{34} \mathrm{H}_{45} \mathrm{~N}_{6}$ requires 537.3706 $[\mathrm{M}+\mathrm{H}]^{+}$. UV $\left(\mathrm{CH}_{2} \mathrm{Cl}_{2}\right) \lambda_{\max } \mathrm{nm}(\varepsilon): 340(8300)$. ${ }^{1} \mathrm{H}$ NMR $\left(\mathrm{CDCl}_{3}\right.$, $298 \mathrm{~K}) \delta_{\mathrm{H}}$ ppm: 1.95 (4H, br.s), 2.21 (4H, br.s), 2.69-2.81 (12H, m), 3.39 (4H, br.s), 3.98 (4H, s), 4.83 (2H, br.s), $6.49\left(2 \mathrm{H}, \mathrm{d},{ }^{3} J=7.8\right.$ $\mathrm{Hz}), 6.97\left(2 \mathrm{H}, \mathrm{d},{ }^{3} J=7.8 \mathrm{~Hz}\right), 7.42\left(2 \mathrm{H}, \mathrm{t},{ }^{3} J_{\text {obs }}=7.6 \mathrm{~Hz}\right), 7.50(2 \mathrm{H}$, $\left.\mathrm{t},{ }^{3} \mathrm{~J}_{o b s}=7.6 \mathrm{~Hz}\right), 7.83\left(2 \mathrm{H}, \mathrm{d},{ }^{3} \mathrm{~J}=8.4 \mathrm{~Hz}\right), 8.04\left(2 \mathrm{H}, \mathrm{d},{ }^{3} \mathrm{~J}=8.4 \mathrm{~Hz}\right)$ (two $\mathrm{NH}$ protons were not assigned). ${ }^{13} \mathrm{C} \mathrm{NMR}\left(\mathrm{CDCl}_{3}, 298 \mathrm{~K}\right) \delta_{\mathrm{c}}$ ppm: 24.6 (2C), 41.8 (2C), 47.1 (4C), 52.5 (4C), 58.7 (2C), 103.4 (2C), 120.7 (2C), 122.8 (2C), 123.5 (2C), 124.2 (2C), 124.5 (2C), $125.8(2 \mathrm{C}), 128.6(2 \mathrm{C}), 133.0(2 \mathrm{C}), 142.9$ (2C).

Macrobicycle 8c. Obtained from compound 5 (304 mg, $0.5 \mathrm{mmol})$ and diamine $7 \mathrm{c}(86 \mathrm{mg}, 0.5 \mathrm{mmol})$. Eluent $\mathrm{CH}_{2} \mathrm{Cl}_{2} /$ $\mathrm{MeOH} 3: 1$. Yield $53 \mathrm{mg}(17 \%)$, yellowish crystalline powder, m.p. 152-154 ${ }^{\circ} \mathrm{C}$. (MALDI-TOF) found: 621.4618. $\mathrm{C}_{40} \mathrm{H}_{57} \mathrm{~N}_{6}$ requires $621.4645[\mathrm{M}+\mathrm{H}]^{+}$. $\mathrm{UV}\left(\mathrm{CH}_{2} \mathrm{Cl}_{2}\right) \lambda_{\text {max }} \mathrm{nm}(\varepsilon)$ : $340(11000)$. ${ }^{1} \mathrm{H}$ NMR $\left(\mathrm{CDCl}_{3}, 298 \mathrm{~K}\right) \delta_{\mathrm{H}} \mathrm{ppm}: 1.40-1.48(8 \mathrm{H}, \mathrm{m}), 1.57(4 \mathrm{H}$, quintet, $\left.{ }^{3} J=6.2 \mathrm{~Hz}\right), 1.80\left(4 \mathrm{H}\right.$, quintet, $\left.{ }^{3} J=6.8 \mathrm{~Hz}\right), 2.55(8 \mathrm{H}$, br.s), $2.82\left(8 \mathrm{H}, \mathrm{t},{ }^{3} J=5.1 \mathrm{~Hz}\right), 3.24\left(4 \mathrm{H}, \mathrm{t},{ }^{3} J=6.5 \mathrm{~Hz}\right), 4.03(4 \mathrm{H}$, s), $6.32\left(2 \mathrm{H}, \mathrm{d},{ }^{3} J=7.8 \mathrm{~Hz}\right), 7.17\left(2 \mathrm{H}, \mathrm{d},{ }^{3} J=7.8 \mathrm{~Hz}\right), 7.42-7.47$ $(2 \mathrm{H}, \mathrm{m}), 7.46-7.51(2 \mathrm{H}, \mathrm{m}), 7.81\left(2 \mathrm{H}, \mathrm{d},{ }^{3} J=8.5 \mathrm{~Hz}\right), 8.14(2 \mathrm{H}$, $\left.\mathrm{d},{ }^{3} \mathrm{~J}=8.5 \mathrm{~Hz}\right)\left(\mathrm{NH}\right.$ protons were not assigned). ${ }^{13} \mathrm{C} \mathrm{NMR}\left(\mathrm{CDCl}_{3}\right.$, $298 \mathrm{~K}) \delta_{\text {c }}$ ppm: $26.8(2 \mathrm{C}), 28.0(2 \mathrm{C}), 28.2(2 \mathrm{C}), 28.6(2 \mathrm{C}), 43.8$ (2C), 47.0 (4C), 52.6 (4C), 59.1 (2C), 103.8 (2C), 120.2 (2C), $122.8(2 \mathrm{C}), 123.6(2 \mathrm{C}), 124.3(2 \mathrm{C}), 124.4(2 \mathrm{C}), 125.9(2 \mathrm{C}), 128.3$ (2C), 132.9 (2C), 143.3 (2C).

Macrobicycle 8d. Obtained from compound 5 (304 mg, 0.5 $\mathrm{mmol})$ and triamine $7 \mathbf{d}(65 \mathrm{mg}, 0.5 \mathrm{mmol})$. Eluent $\mathrm{CH}_{2} \mathrm{Cl}_{2} / \mathrm{MeOH} /$ $\mathrm{NH}_{3}$ aq 100:20:1. Yield $52 \mathrm{mg}$ (18\%), yellowish crystalline powder, m.p. $158-160{ }^{\circ} \mathrm{C}$. (MALDI-TOF) found: 580.4087. $\mathrm{C}_{36} \mathrm{H}_{50} \mathrm{~N}_{7}$ requires $580.4128[\mathrm{M}+\mathrm{H}]^{+}$. $\mathrm{UV}\left(\mathrm{CH}_{2} \mathrm{Cl}_{2}\right) \lambda_{\max } \mathrm{nm}(\varepsilon)$ : $339(13000)$. ${ }^{1} \mathrm{H}$ NMR $\left(\mathrm{CDCl}_{3}, 298 \mathrm{~K}\right) \delta_{\mathrm{H}} \mathrm{ppm}: 1.96\left(4 \mathrm{H}\right.$, quintet, $\left.{ }^{3} J=5.0 \mathrm{~Hz}\right)$, 2.40 (4H, br.s), $2.66(12 \mathrm{H}$, br.s $), 2.91\left(4 \mathrm{H}, \mathrm{t},{ }^{3} J=5.0 \mathrm{~Hz}\right), 3.21(4 \mathrm{H}$, $\left.\mathrm{t},{ }^{3} \mathrm{~J}=6.4 \mathrm{~Hz}\right), 3.89(4 \mathrm{H}, \mathrm{s}), 5.82\left(2 \mathrm{H}, \mathrm{d},{ }^{3} \mathrm{~J}=7.8 \mathrm{~Hz}\right), 6.94(2 \mathrm{H}, \mathrm{d}$, $\left.{ }^{3} J=7.8 \mathrm{~Hz}\right), 7.38\left(2 \mathrm{H}, \mathrm{t},{ }^{3} J_{o b s}=7.5 \mathrm{~Hz}\right), 7.49\left(2 \mathrm{H}, \mathrm{t},{ }^{3} J_{o b s}=7.6 \mathrm{~Hz}\right)$, $7.88\left(2 \mathrm{H}, \mathrm{d},{ }^{3} \mathrm{~J}=8.5 \mathrm{~Hz}\right), 8.10\left(2 \mathrm{H}, \mathrm{d},{ }^{3} J=8.5 \mathrm{~Hz}\right)$ (NH protons were not assigned). ${ }^{13} \mathrm{C}$ NMR $\left(\mathrm{CDCl}_{3}, 298 \mathrm{~K}\right) \delta_{\mathrm{c}} \mathrm{ppm}: 28.6(2 \mathrm{C})$, 44.6 (2C), 46.4 (4C), 49.3 (2C), 52.7 (4C), 58.9 (2C), 104.0 (2C), 120.9 (2C), 122.7 (2C), 123.7 (2C), 123.9 (2C), 124.2 (2C), 125.7 (2C), 129.1 (2C), 132.7 (2C), 143.6 (2C).

Cyclodimer 10d. Obtained as the second product in the synthesis of macrobicycle 8d. Eluent $\mathrm{CH}_{2} \mathrm{Cl}_{2} / \mathrm{MeOH} / \mathrm{NH}_{3}$ aq 100:20:2. Yield $55 \mathrm{mg}(19 \%)$, beige glassy compound. (MALDITOF) found: 1159.67. $\mathrm{C}_{72} \mathrm{H}_{99} \mathrm{~N}_{14}$ requires $1159.82[\mathrm{M}+\mathrm{H}]^{+} .{ }^{1} \mathrm{H} \mathrm{NMR}$ $\left(\mathrm{CDCl}_{3}, 298 \mathrm{~K}\right) \delta_{\mathrm{H}}$ ppm: $1.98(8 \mathrm{H}$, br.s $), 2.51-2.69(32 \mathrm{H}, \mathrm{m}), 2.87$ (8H, br.s), 3.34 (8H, br.s), 3.91 (8H, br.s), 6.43 (4H, d, $\left.{ }^{3} J=7.7 \mathrm{~Hz}\right)$, $7.23\left(4 \mathrm{H}, \mathrm{d},{ }^{3} \mathrm{~J}=7.7 \mathrm{~Hz}\right), 7.29-7.46(8 \mathrm{H}, \mathrm{m}), 7.84$ (4H, br.s), 8.09 (4H, br.s) (NH protons were not assigned).
Macrobicycle 8e. Obtained from compound 5 (304 mg, 0.5 mmol) and tetraamine 7 e ( $80 \mathrm{mg}, 0.5 \mathrm{mmol})$. Eluent $\mathrm{CH}_{2} \mathrm{Cl}_{2} / \mathrm{MeOH} /$ $\mathrm{NH}_{3}$ aq 100:20:2. Yield $28 \mathrm{mg}$ (9\%), yellowish glassy compound. (MALDI-TOF) found: 609.4367. $\mathrm{C}_{37} \mathrm{H}_{53} \mathrm{~N}_{8}$ requires 609.4393 $[\mathrm{M}+\mathrm{H}]^{+}$. UV $\left(\mathrm{CH}_{2} \mathrm{Cl}_{2}\right) \lambda_{\max } \mathrm{nm}(\varepsilon): 339(10000) .{ }^{1} \mathrm{H} \mathrm{NMR}\left(\mathrm{CDCl}_{3}\right.$, $298 \mathrm{~K}) \delta_{\mathrm{H}}$ ppm: 1.82 (2H, br.s), 2.47 (4H, br.s), 2.68-2.95 (20H, m), 3.17 (4H, br.s), 3.92 (4H, s), $5.81\left(2 \mathrm{H}, \mathrm{d},{ }^{3} J=7.7 \mathrm{~Hz}\right), 6.97(2 \mathrm{H}, \mathrm{d}$, $\left.{ }^{3} J=7.7 \mathrm{~Hz}\right), 7.39-7.44(2 \mathrm{H}, \mathrm{m}), 7.50-7.55(2 \mathrm{H}, \mathrm{m}), 7.92\left(2 \mathrm{H}, \mathrm{d},{ }^{3} J\right.$ $=8.2 \mathrm{~Hz}), 8.08\left(2 \mathrm{H}, \mathrm{d},{ }^{3} J=8.2 \mathrm{~Hz}\right)(\mathrm{NH}$ protons were not assigned $)$. ${ }^{13} \mathrm{C} \mathrm{NMR}\left(\mathrm{CDCl}_{3}, 298 \mathrm{~K}\right) \delta_{\mathrm{c}} \mathrm{ppm}: 31.8(1 \mathrm{C}), 41.7$ (2C), 43.9 (2C), 47.3 (4C), 51.8 (4C), 53.5 (2C), 58.7 (2C), 104.4 (2C), 120.8 (2C), 122.9 (2C), 123.4 (2C), 123.8 (2C), 124.4 (2C), 126.0 (2C), 129.2 (2C), 133.0 (2C), 143.3 (2C).

Cyclodimer 10e. Obtained as the second product in the synthesis of macrobicycle 8e. Eluent $\mathrm{CH}_{2} \mathrm{Cl}_{2} / \mathrm{MeOH} / \mathrm{NH}_{3}$ aq 100:20:2. Yield $37 \mathrm{mg}$ (12\%), yellowish glassy compound. (MALDI-TOF) found: 1217.91. $\mathrm{C}_{74} \mathrm{H}_{105} \mathrm{~N}_{16}$ requires $1217.87[\mathrm{M}+\mathrm{H}]^{+}$. UV $\left(\mathrm{CH}_{2} \mathrm{Cl}_{2}\right) \lambda_{\max }$ $\mathrm{nm}(\varepsilon): 339$ (20000). ${ }^{1} \mathrm{H}$ NMR $\left(\mathrm{CDCl}_{3}, 298 \mathrm{~K}\right) \delta_{\mathrm{H}} \mathrm{ppm}: 1.75(4 \mathrm{H}$, quintet, $\left.{ }^{3} J=5.6 \mathrm{~Hz}\right), 2.45-2.71(32 \mathrm{H}, \mathrm{m}), 2.87\left(8 \mathrm{H}, \mathrm{t},{ }^{3} J=5.5 \mathrm{~Hz}\right)$, $3.02\left(8 \mathrm{H}, \mathrm{t},{ }^{3} \mathrm{~J}=5.8 \mathrm{~Hz}\right), 3.24\left(8 \mathrm{H}, \mathrm{t},{ }^{3} \mathrm{~J}=5.4 \mathrm{~Hz}\right), 3.90(8 \mathrm{H}, \mathrm{s})$, 4.76 (4H, br.s), $6.03\left(4 \mathrm{H}, \mathrm{d},{ }^{3} J=7.8 \mathrm{~Hz}\right), 7.05\left(4 \mathrm{H}, \mathrm{d},{ }^{3} J=7.8 \mathrm{~Hz}\right)$, $7.40\left(4 \mathrm{H}, \mathrm{t},{ }^{3} \mathrm{~J}_{o b s}=7.5 \mathrm{~Hz}\right), 7.48\left(4 \mathrm{H}, \mathrm{t},{ }^{3} \mathrm{~J}_{o b s}=7.8 \mathrm{~Hz}\right), 7.89(4 \mathrm{H}, \mathrm{d}$, ${ }^{3} J=8.0 \mathrm{~Hz}$ ), $8.12\left(4 \mathrm{H}, \mathrm{d},{ }^{3} \mathrm{~J}=8.4 \mathrm{~Hz}\right.$ ) (four $\mathrm{NH}$ protons were not assigned). ${ }^{13} \mathrm{C} \mathrm{NMR}\left(\mathrm{CDCl}_{3}, 298 \mathrm{~K}\right) \delta_{\mathrm{c}} \mathrm{ppm}$ : $28.9(2 \mathrm{C}), 43.8(4 \mathrm{C})$, 45.9 (8C), 48.8 (4C), 49.4 (4C), 53.2 (8C), 59.3 (4C), 104.6 (4C), 120.6 (4C), 123.5 (4C), 123.9 (4C), 124.1 (4C), 124.4 (4C), 125.8 (4C), 128.4 (4C), 132.8 (4C), 143.1 (4C).

Macrobicycle 8f. Obtained from compound 5 (304 mg, 0.5 mmol) and tetraamine $7 \mathbf{f}(87 \mathrm{mg}, 0.5 \mathrm{mmol})$. Eluent $\mathrm{CH}_{2} \mathrm{Cl}_{2} / \mathrm{MeOH} /$ $\mathrm{NH}_{3}$ aq 100:20:1. Yield $40 \mathrm{mg}$ (13\%), beige glassy compound. (MALDI-TOF) found: 622.4504. $\mathrm{C}_{38} \mathrm{H}_{55} \mathrm{~N}_{8}$ requires 623.4550 $[\mathrm{M}+\mathrm{H}]^{+}$. UV $\left(\mathrm{CH}_{2} \mathrm{Cl}_{2}\right) \lambda_{\max } \mathrm{nm}(\varepsilon): 340(10000) .{ }^{1} \mathrm{H} \mathrm{NMR}\left(\mathrm{CDCl}_{3}\right.$, $298 \mathrm{~K}) \delta_{\mathrm{H}}$ ppm:1.95 (4H, quintet, $\left.{ }^{3} J=5.6 \mathrm{~Hz}\right), 2.45-2.80(16 \mathrm{H}, \mathrm{m})$, $2.85\left(4 \mathrm{H}, \mathrm{t},{ }^{3} \mathrm{~J}=5.2 \mathrm{~Hz}\right), 3.04(4 \mathrm{H}, \mathrm{s}), 3.34\left(4 \mathrm{H}, \mathrm{t},{ }^{3} \mathrm{~J}=6.3 \mathrm{~Hz}\right), 3.95$ $(4 \mathrm{H}, \mathrm{s}), 6.24\left(2 \mathrm{H}, \mathrm{d},{ }^{3} J=7.8 \mathrm{~Hz}\right), 7.15\left(2 \mathrm{H}, \mathrm{d},{ }^{3} J=7.8 \mathrm{~Hz}\right), 7.49$ $\left(2 \mathrm{H}, \mathrm{t},{ }^{3} J_{o b s}=7.8 \mathrm{~Hz}\right), 7.56\left(2 \mathrm{H}, \mathrm{t},{ }^{3} J_{o b s}=8.1 \mathrm{~Hz}\right), 7.95\left(2 \mathrm{H}, \mathrm{d},{ }^{3} \mathrm{~J}=\right.$ $8.5 \mathrm{~Hz}), 8.14\left(2 \mathrm{H}, \mathrm{d},{ }^{3} J=8.9 \mathrm{~Hz}\right)$ (NH protons were not assigned). ${ }^{13} \mathrm{C} \mathrm{NMR}\left(\mathrm{CDCl}_{3}, 298 \mathrm{~K}\right) \delta_{\mathrm{c}} \mathrm{ppm}: 28.7(2 \mathrm{C}), 44.3(2 \mathrm{C}), 46.2(4 \mathrm{C})$, 49.4 (2C), 50.1 (2C), 52.1 (4C), 59.6 (2C), 103.5 (2C), 120.8 (2C), 122.4 (2C), 123.7 (2C), 124.0 (2C), 124.3 (2C), 125.7 (2C), 128.1 (2C), 133.1 (2C), 143.7 (2C).

Cyclodimer 10f. Obtained as the second product in the synthesis of macrobicycle 8f. Eluent $\mathrm{CH}_{2} \mathrm{Cl}_{2} / \mathrm{MeOH} / \mathrm{NH}_{3}$ aq 100:20:1. Yield $19 \mathrm{mg}(6 \%)$, yellowish glassy compound. (MALDI-TOF) found: 1245.74. $\mathrm{C}_{76} \mathrm{H}_{109} \mathrm{~N}_{16}$ requires $1245.90[\mathrm{M}+\mathrm{H}]^{+}$. UV $\left(\mathrm{CH}_{2} \mathrm{Cl}_{2}\right) \lambda_{\max }$ $\mathrm{nm}(\varepsilon): 340$ (20000). ${ }^{1} \mathrm{H}$ NMR $\left(\mathrm{CDCl}_{3}, 298 \mathrm{~K}\right) \delta_{\mathrm{H}} \mathrm{ppm}: 1.93(8 \mathrm{H}$, quintet, $\left.{ }^{3} \mathrm{~J}=5.6 \mathrm{~Hz}\right), 2.45-2.75(32 \mathrm{H}, \mathrm{m}), 2.88(8 \mathrm{H}, \mathrm{s}), 2.91(8 \mathrm{H}, \mathrm{t}$, $\left.{ }^{3} J=5.6 \mathrm{~Hz}\right), 3.32\left(8 \mathrm{H}, \mathrm{t},{ }^{3} \mathrm{~J}=6.3 \mathrm{~Hz}\right), 3.91(8 \mathrm{H}, \mathrm{s}), 6.29\left(4 \mathrm{H}, \mathrm{d},{ }^{3} J=\right.$ $7.8 \mathrm{~Hz}), 7.19\left(4 \mathrm{H}, \mathrm{d},{ }^{3} J=7.8 \mathrm{~Hz}\right), 7.35-7.44(8 \mathrm{H}, \mathrm{m}), 7.86\left(4 \mathrm{H}, \mathrm{d},{ }^{3} J\right.$ $=7.9 \mathrm{~Hz}), 8.11\left(4 \mathrm{H}, \mathrm{d},{ }^{3} \mathrm{~J}=9.1 \mathrm{~Hz}\right)(\mathrm{NH}$ protons were not assigned $)$. ${ }^{13} \mathrm{C} \mathrm{NMR}\left(\mathrm{CDCl}_{3}, 298 \mathrm{~K}\right) \delta_{\mathrm{c}} \mathrm{ppm}: 27.1(4 \mathrm{C}), 44.3(4 \mathrm{C}), 46.3(8 \mathrm{C})$, 50.3 (4C), 51.9 (4C), 52.7 (8C), 59.5 (4C), 103.6 (4C), $121.1(4 \mathrm{C})$, 123.8 (4C), 124.0 (4C), 124.1 (4C), 124.3 (4C), 125.8 (4C), 128.7 (4C), 132.8 (4C), 144.1 (4C).

Macrobicycle 8g. Obtained from compound 5 (304 mg, 0.5 mmol) and tetraamine $7 \mathbf{g}(94 \mathrm{mg}, 0.5 \mathrm{mmol})$. Eluent $\mathrm{CH}_{2} \mathrm{Cl}_{2} / \mathrm{MeOH} /$ $\mathrm{NH}_{3}$ aq 100:20:2. Yield $55 \mathrm{mg}(17 \%)$, beige glassy compound. (MALDI-TOF) found: 637.4753. $\mathrm{C}_{39} \mathrm{H}_{57} \mathrm{~N}_{8}$ requires 637.4706 $[\mathrm{M}+\mathrm{H}]^{+}$. UV $\left(\mathrm{CH}_{2} \mathrm{Cl}_{2}\right) \lambda_{\max } \mathrm{nm}(\varepsilon): 340(12000) .{ }^{1} \mathrm{H} \mathrm{NMR}\left(\mathrm{CDCl}_{3}\right.$, $298 \mathrm{~K}) \delta_{\mathrm{H}}$ ppm: $1.84\left(4 \mathrm{H}\right.$, quintet, $\left.{ }^{3} \mathrm{~J}=6.8 \mathrm{~Hz}\right), 1.96\left(2 \mathrm{H}\right.$, quintet, ${ }^{3} \mathrm{~J}$ $=6.5 \mathrm{~Hz}), 2.45-2.90(20 \mathrm{H}, \mathrm{m}), 2.94\left(4 \mathrm{H}, \mathrm{t},{ }^{3} J=6.5 \mathrm{~Hz}\right), 3.32(4 \mathrm{H}, \mathrm{t}$, $\left.{ }^{3} J=6.2 \mathrm{~Hz}\right), 3.92(4 \mathrm{H}, \mathrm{s}), 6.09\left(2 \mathrm{H}, \mathrm{d},{ }^{3} J=7.8 \mathrm{~Hz}\right), 7.06\left(2 \mathrm{H}, \mathrm{d},{ }^{3} J\right.$ $=7.8 \mathrm{~Hz}), 7.39-7.46(4 \mathrm{H}, \mathrm{m}), 7.88\left(2 \mathrm{H}, \mathrm{d},{ }^{3} J=8.3 \mathrm{~Hz}\right), 8.13(2 \mathrm{H}, \mathrm{d}$, $\left.{ }^{3} J=7.9 \mathrm{~Hz}\right)\left(\mathrm{NH}\right.$ protons were not assigned). ${ }^{13} \mathrm{C} \mathrm{NMR}\left(\mathrm{CDCl}_{3}, 298\right.$ K) $\delta_{c}$ ppm: 25.1 (1C), $30.4(2 \mathrm{C}), 44.6(2 \mathrm{C}), 46.2$ (4C), 53.1 (4C), $55.4(2 \mathrm{C}), 57.6(2 \mathrm{C}), 59.8(2 \mathrm{C}), 104.0(2 \mathrm{C}), 121.5(2 \mathrm{C}), 123.4(2 \mathrm{C})$, $123.8(2 \mathrm{C}), 124.1(2 \mathrm{C}), 124.2(2 \mathrm{C}), 126.0$ (2C), $128.6(2 \mathrm{C}), 133.1$ (2C), 143.9 (2C). 
Cyclodimer 10g. Obtained as the second product in the synthesis of macrobicycle 8g. Eluent $\mathrm{CH}_{2} \mathrm{Cl}_{2} / \mathrm{MeOH} / \mathrm{NH}_{3}$ aq 100:20:2. Yield $12 \mathrm{mg}$ (4\%), yellowish glassy compound. (MALDITOF) found: 1273.81. $\mathrm{C}_{78} \mathrm{H}_{113} \mathrm{~N}_{16}$ requires $1273.93[\mathrm{M}+\mathrm{H}]^{+}$. UV $\left(\mathrm{CH}_{2} \mathrm{Cl}_{2}\right) \lambda_{\max } \mathrm{nm}(\varepsilon): 339$ (23000). ${ }^{1} \mathrm{H}$ NMR $\left(\mathrm{CDCl}_{3}, 298 \mathrm{~K}\right) \delta_{\mathrm{H}}$ ppm: $1.84\left(4 \mathrm{H}\right.$, quintet, $\left.{ }^{3} J=6.8 \mathrm{~Hz}\right), 1.91\left(8 \mathrm{H}\right.$, quintet, ${ }^{3} J=5.9$ $\mathrm{Hz}), 2.45-2.72(32 \mathrm{H}, \mathrm{m}), 2.77\left(8 \mathrm{H}, \mathrm{t},{ }^{3} \mathrm{~J}=6.8 \mathrm{~Hz}\right), 2.87\left(8 \mathrm{H}, \mathrm{t},{ }^{3} \mathrm{~J}\right.$ $=5.7 \mathrm{~Hz}), 3.32\left(8 \mathrm{H}, \mathrm{t},{ }^{3} \mathrm{~J}=6.2 \mathrm{~Hz}\right), 3.89(8 \mathrm{H}, \mathrm{s}), 6.34\left(4 \mathrm{H}, \mathrm{d},{ }^{3} \mathrm{~J}=\right.$ $7.8 \mathrm{~Hz}), 7.20\left(4 \mathrm{H}, \mathrm{d},{ }^{3} \mathrm{~J}=7.8 \mathrm{~Hz}\right), 7.30\left(4 \mathrm{H}, \mathrm{t},{ }^{3} J_{o b s}=7.6 \mathrm{~Hz}\right), 7.37$ $\left(4 \mathrm{H}, \mathrm{t},{ }^{3} \mathrm{~J}_{o b s}=8.0 \mathrm{~Hz}\right), 7.88\left(4 \mathrm{H}, \mathrm{d},{ }^{3} J=8.3 \mathrm{~Hz}\right), 8.10\left(4 \mathrm{H}, \mathrm{d},{ }^{3} \mathrm{~J}=\right.$ $8.1 \mathrm{~Hz}$ ) (NH protons were not assigned). ${ }^{13} \mathrm{C} \mathrm{NMR}\left(\mathrm{CDCl}_{3}, 298\right.$ K) $\delta$ ppm: 25.3 (2C), $28.6(4 \mathrm{C}), 43.9$ (4C), 45.9 (8C), 48.8 (4C), 49.1 (4C), 52.9 (8C), 59.6 (4C), 103.3 (4C), 120.9 (4C), 122.5 (4C), 123.8 (4C), 124.0 (4C), 124.1 (4C), 125.8 (4C), 128.2 (4C), 132.9 (4C), 143.8 (4C).

Macrobicycle 8h. Obtained from compound 5 (304 mg, 0.5 mmol) and dioxadiamine $7 \mathbf{h}$ (74 $\mathrm{mg}, 0.5 \mathrm{mmol})$. Eluent $\mathrm{CH}_{2} \mathrm{Cl}_{2}$ / $\mathrm{MeOH} 3: 1$. Yield $32 \mathrm{mg}$ (11\%), beige crystalline powder. (MALDITOF) found: $597.3867 . \mathrm{C}_{36} \mathrm{H}_{49} \mathrm{~N}_{6} \mathrm{O}_{2}$ requires $597.3917[\mathrm{M}+\mathrm{H}]^{+}$. UV $\left(\mathrm{CH}_{2} \mathrm{Cl}_{2}\right) \lambda_{\max } \mathrm{nm}(\varepsilon): 337$ (12000). ${ }^{1} \mathrm{H} \mathrm{NMR}\left(\mathrm{CDCl}_{3}, 298 \mathrm{~K}\right) \delta_{\mathrm{H}}$ ppm: 2.53 (8H, br.s), 2.69 (8H, br.s), 3.34 (4H, t, $\left.{ }^{3} J=5.9 \mathrm{~Hz}\right), 3.75$ $(4 \mathrm{H}, \mathrm{s}), 3.86\left(4 \mathrm{H}, \mathrm{t},{ }^{3} J=6.0 \mathrm{~Hz}\right), 3.94(4 \mathrm{H}, \mathrm{s}), 4.66$ (2H, br.s), 6.05 $\left(2 \mathrm{H}, \mathrm{d},{ }^{3} J=7.8 \mathrm{~Hz}\right), 7.00\left(2 \mathrm{H}, \mathrm{d},{ }^{3} J=7.8 \mathrm{~Hz}\right), 7.39-7.44(2 \mathrm{H}, \mathrm{m})$, $7.48-7.53(2 \mathrm{H}, \mathrm{m}), 7.91\left(2 \mathrm{H}, \mathrm{d},{ }^{3} J=8.2 \mathrm{~Hz}\right), 8.13\left(2 \mathrm{H}, \mathrm{d},{ }^{3} J=8.4\right.$ $\mathrm{Hz}$ ) (two $\mathrm{NH}$ protons were not assigned). ${ }^{13} \mathrm{C} \mathrm{NMR}\left(\mathrm{CDCl}_{3}, 298 \mathrm{~K}\right)$ $\delta_{c}$ ppm: 43.9 (2C), $46.6(4 \mathrm{C}), 52.9$ (4C), 59.3 (2C), 69.3 (2C), 70.4 (2C), 104.7 (2C), 120.8 (2C), 123.8 (2C), 124.1 (4C), 124.5 (2C), 125.9 (2C), $128.6(2 \mathrm{C}), 132.8(2 \mathrm{C}), 143.0(2 \mathrm{C})$.

Cyclodimer 10h. Obtained as the second product in the synthesis of macrobicycle $\mathbf{8 h}$. Eluent $\mathrm{CH}_{2} \mathrm{Cl}_{2} / \mathrm{MeOH} / \mathrm{NH}_{3} \mathrm{aq}$ 100:20:1-100:20:2. Yield $18 \mathrm{mg}$ (6\%), yellowish glassy compound. (MALDI-TOF) found: 1193.59. $\mathrm{C}_{72} \mathrm{H}_{97} \mathrm{~N}_{12} \mathrm{O}_{4}$ requires 1193.78 $[\mathrm{M}+\mathrm{H}]^{+}$. UV $\left(\mathrm{CH}_{2} \mathrm{Cl}_{2}\right) \lambda_{\max } \mathrm{nm}(\varepsilon): 336(23000) .{ }^{1} \mathrm{H} \mathrm{NMR}\left(\mathrm{CDCl}_{3}\right.$, $298 \mathrm{~K}) \delta_{\mathrm{H}}$ ppm: $2.50-2.65(32 \mathrm{H}, \mathrm{m}), 3.41\left(8 \mathrm{H}, \mathrm{t},{ }^{3} \mathrm{~J}=5.5 \mathrm{~Hz}\right), 3.73$ $(8 \mathrm{H}, \mathrm{s}), 3.85\left(8 \mathrm{H}, \mathrm{t},{ }^{3} \mathrm{~J}=5.5 \mathrm{~Hz}\right), 3.92(8 \mathrm{H}, \mathrm{s}), 4.86$ (4H, br.s), 6.40 $\left(4 \mathrm{H}, \mathrm{d},{ }^{3} J=7.8 \mathrm{~Hz}\right), 7.20\left(4 \mathrm{H}, \mathrm{d},{ }^{3} J=7.8 \mathrm{~Hz}\right), 7.31\left(4 \mathrm{H}, \mathrm{t},{ }^{3} J_{o b s}=7.9\right.$ $\mathrm{Hz}), 7.34-7.39(4 \mathrm{H}, \mathrm{m}), 7.83\left(4 \mathrm{H}, \mathrm{d},{ }^{3} J=8.3 \mathrm{~Hz}\right), 8.06\left(4 \mathrm{H}, \mathrm{d},{ }^{3} J=\right.$ $8.0 \mathrm{~Hz}$ ) (four $\mathrm{NH}$ protons were not assigned).

Macrobicycle 8i. Obtained from compound 5 (304 mg, 0.5 mmol) and dioxadiamine $7 \mathbf{i}(102 \mathrm{mg}, 0.5 \mathrm{mmol})$. Eluent $\mathrm{CH}_{2} \mathrm{Cl}_{2} /$ $\mathrm{MeOH} 3: 1$. Yield $87 \mathrm{mg}$ (26\%), beige crystalline powder, m.p. 143-145 ${ }^{\circ} \mathrm{C}$. (MALDI-TOF) found: $653.4507 . \mathrm{C}_{40} \mathrm{H}_{57} \mathrm{~N}_{6} \mathrm{O}_{2}$ requires $653.4543[\mathrm{M}+\mathrm{H}]^{+}$. UV $\left(\mathrm{CH}_{2} \mathrm{Cl}_{2}\right) \lambda_{\max } \mathrm{nm}(\varepsilon): 340(10000) .{ }^{1} \mathrm{H} \mathrm{NMR}$ $\left(\mathrm{CDCl}_{3}, 298 \mathrm{~K}\right) \delta_{\mathrm{H}} \mathrm{ppm}: 1.82-1.86(4 \mathrm{H}, \mathrm{m}), 2.06\left(4 \mathrm{H}\right.$, quintet, ${ }^{3} J=$ $5.9 \mathrm{~Hz}), 2.55$ (8H, br.s), 2.67 (8H, br.s), $3.39\left(4 \mathrm{H}, \mathrm{t},{ }^{3} \mathrm{~J}=6.5 \mathrm{~Hz}\right)$, 3.53-3.57 (4H, m), $3.70\left(4 \mathrm{H}, \mathrm{t},{ }^{3} J=5.2 \mathrm{~Hz}\right), 3.90(4 \mathrm{H}, \mathrm{s}), 5.09(2 \mathrm{H}$, br.s), $6.43\left(2 \mathrm{H}, \mathrm{d},{ }^{3} J=7.8 \mathrm{~Hz}\right), 7.20\left(2 \mathrm{H}, \mathrm{d},{ }^{3} J=7.8 \mathrm{~Hz}\right), 7.38-7.43$ $(2 \mathrm{H}, \mathrm{m}), 7.43-7.48(2 \mathrm{H}, \mathrm{m}), 7.85\left(2 \mathrm{H}, \mathrm{d},{ }^{3} J=8.0 \mathrm{~Hz}\right), 8.11(2 \mathrm{H}, \mathrm{d}$, ${ }^{3} \mathrm{~J}=8.0 \mathrm{~Hz}$ ) (two $\mathrm{NH}$ protons were not assigned). ${ }^{13} \mathrm{C} \mathrm{NMR}\left(\mathrm{CDCl}_{3}\right.$, $298 \mathrm{~K}) \delta_{\mathrm{c}}$ ppm: 27.1 (2C), 29.0 (2C), 43.3 (2C), 48.3 (4C), 52.2 (4C), 60.0 (2C), 70.8 (2C), $71.5(2 \mathrm{C}), 102.7$ (2C), 120.7 (2C), 122.0 (2C), 123.5 (2C), 124.2 (2C), 124.3 (2C), 126.0 (2C), 128.6 (2C), 132.8 (2C), 143.8 (2C).

Cyclodimer 10i. Obtained as the second product in the synthesis of macrobicycle 8i. Eluent $\mathrm{CH}_{2} \mathrm{Cl}_{2} / \mathrm{MeOH} / \mathrm{NH}_{3}$ aq 100:20:1. Yield $53 \mathrm{mg}(16 \%)$, beige glassy compound. (MALDITOF) found: 1305.66. $\mathrm{C}_{80} \mathrm{H}_{113} \mathrm{~N}_{12} \mathrm{O}_{4}$ requires $1305.90[\mathrm{M}+\mathrm{H}]^{+} .{ }^{1} \mathrm{H}$ $\operatorname{NMR}\left(\mathrm{CDCl}_{3}, 298 \mathrm{~K}\right) \delta_{\mathrm{H}} \mathrm{ppm}: 1.73-1.77(8 \mathrm{H}, \mathrm{m}), 2.01(8 \mathrm{H}$, quintet, $\left.{ }^{3} \mathrm{~J}=5.6 \mathrm{~Hz}\right), 2.52-2.70(32 \mathrm{H}, \mathrm{m}), 3.30(8 \mathrm{H}, \mathrm{br} . \mathrm{s}), 3.45-3.50(8 \mathrm{H}, \mathrm{m})$, $3.61\left(8 \mathrm{H}, \mathrm{t},{ }^{3} \mathrm{~J}=5.4 \mathrm{~Hz}\right), 3.88(8 \mathrm{H}, \mathrm{s}), 5.12(4 \mathrm{H}, \mathrm{br} . \mathrm{s}), 6.41(4 \mathrm{H}, \mathrm{d}$, $\left.{ }^{3} J=7.8 \mathrm{~Hz}\right), 7.22\left(4 \mathrm{H}, \mathrm{d},{ }^{3} J=7.8 \mathrm{~Hz}\right), 7.34-7.39(8 \mathrm{H}, \mathrm{m}), 7.89(4 \mathrm{H}$, $\mathrm{d},{ }^{3} \mathrm{~J}=9.0 \mathrm{~Hz}$ ), $8.06\left(4 \mathrm{H}, \mathrm{d},{ }^{3} \mathrm{~J}=8.9 \mathrm{~Hz}\right.$ ) (four $\mathrm{NH}$ protons were not assigned). ${ }^{13} \mathrm{C} \mathrm{NMR}\left(\mathrm{CDCl}_{3}, 298 \mathrm{~K}\right) \delta_{\mathrm{c}} \mathrm{ppm}: 26.6(4 \mathrm{C}), 28.9(4 \mathrm{C})$, 43.0 (4C), 45.9 (8C), 52.2 (8C), 58.5 (4C), 70.7 (4C), 71.1 (4C), 103.2 (4C), 120.6 (4C), 122.2 (4C), 123.8 (4C), 124.1 (4C), 124.3 (4C), 125.8 (4C), 128.7 (4C), 133.0 (4C), 143.7 (4C).

Macrobicycle $8 \mathbf{j}$. Obtained from compound 5 (304 mg, 0.5 mmol) and trioxadiamine $7 \mathbf{j}(110 \mathrm{mg}, 0.5 \mathrm{mmol})$. Eluent $\mathrm{CH}_{2} \mathrm{Cl}_{2} /$
MeOH 3:1. Yield $78 \mathrm{mg}$ (23\%), beige glassy compound. (MALDITOF) found: 669.4468. $\mathrm{C}_{40} \mathrm{H}_{57} \mathrm{~N}_{6} \mathrm{O}_{3}$ requires $669.4492[\mathrm{M}+\mathrm{H}]^{+}$. UV $\left(\mathrm{CH}_{2} \mathrm{Cl}_{2}\right) \lambda_{\max } \mathrm{nm}(\varepsilon): 340$ (12000). ${ }^{1} \mathrm{H} \mathrm{NMR}\left(\mathrm{CDCl}_{3}, 298 \mathrm{~K}\right) \delta_{\mathrm{H}}$ ppm: 2.05 (4H, quintet, $\left.{ }^{3} \mathrm{~J}=5.8 \mathrm{~Hz}\right), 2.56(8 \mathrm{H}$, br.s), $2.66(8 \mathrm{H}$, br.s $)$, $3.37\left(4 \mathrm{H}, \mathrm{t},{ }^{3} J=6.2 \mathrm{~Hz}\right), 3.67-3.70(4 \mathrm{H}, \mathrm{m}), 3.71\left(4 \mathrm{H}, \mathrm{t},{ }^{3} J=5.3\right.$ $\mathrm{Hz}), 3.75-3.78(4 \mathrm{H}, \mathrm{m}), 3.90(4 \mathrm{H}, \mathrm{s}), 5.03(2 \mathrm{H}, \mathrm{br} . \mathrm{s}), 6.33\left(2 \mathrm{H}, \mathrm{d},{ }^{3} \mathrm{~J}\right.$ $=7.8 \mathrm{~Hz}), 7.18\left(2 \mathrm{H}, \mathrm{d},{ }^{3} \mathrm{~J}=7.8 \mathrm{~Hz}\right), 7.35-7.40(2 \mathrm{H}, \mathrm{m}), 7.41-7.46$ $(2 \mathrm{H}, \mathrm{m}),, 7.88\left(2 \mathrm{H}, \mathrm{d},{ }^{3} J=8.3 \mathrm{~Hz}\right), 8.11\left(2 \mathrm{H}, \mathrm{d},{ }^{3} J=8.2 \mathrm{~Hz}\right.$ ) (two $\mathrm{NH}$ protons were not assigned). ${ }^{13} \mathrm{C} \mathrm{NMR}\left(\mathrm{CDCl}_{3}, 298 \mathrm{~K}\right) \delta_{c} \mathrm{ppm}$ : 28.9 (2C), 42.9 (2C), 46.2 (4C), 52.7 (4C), 59.2 (2C), 70.4 (2C), 70.7 (4C), 103.6 (2C), 120.7 (2C), 122.6 (2C), 123.7 (2C), 124.0 (2C), 124.2 (2C), 125.8 (2C), 128.3 (2C), 132.8 (2C), $143.5(2 \mathrm{C})$.

Cyclodimer 10j. Obtained as the second product in the synthesis of macrobicycle 8j. Eluent $\mathrm{CH}_{2} \mathrm{Cl}_{2} / \mathrm{MeOH} / \mathrm{NH}_{3}$ aq 100:20:1. Yield $19 \mathrm{mg}(6 \%)$, yellowish glassy compound. (MALDI-TOF) found: 1337.71. $\mathrm{C}_{80} \mathrm{H}_{113} \mathrm{~N}_{12} \mathrm{O}_{6}$ requires $1337.89[\mathrm{M}+\mathrm{H}]^{+}$. UV $\left(\mathrm{CH}_{2} \mathrm{Cl}_{2}\right) \lambda_{\text {max }}$ $\mathrm{nm}(\varepsilon): 340$ (23000). ${ }^{1} \mathrm{H}$ NMR $\left(\mathrm{CDCl}_{3}, 298 \mathrm{~K}\right) \delta_{\mathrm{H}} \mathrm{ppm}: 1.98(8 \mathrm{H}$, quintet, $\left.{ }^{3} J=5.7 \mathrm{~Hz}\right), 2.48-2.70(32 \mathrm{H}, \mathrm{m}), 3.32\left(8 \mathrm{H}, \mathrm{t},{ }^{3} J=5.4 \mathrm{~Hz}\right)$, 3.56-3.61 (8H, m), $3.62\left(8 \mathrm{H}, \mathrm{t},{ }^{3} \mathrm{~J}=5.4 \mathrm{~Hz}\right), 3.66-3.70(8 \mathrm{H}, \mathrm{m})$, $3.88(8 \mathrm{H}, \mathrm{s}), 5.07$ (4H, br.s), $6.41\left(4 \mathrm{H}, \mathrm{d},{ }^{3} J=7.7 \mathrm{~Hz}\right), 7.22(4 \mathrm{H}$, $\left.\mathrm{d},{ }^{3} \mathrm{~J}=7.7 \mathrm{~Hz}\right), 7.32-7.41(8 \mathrm{H}, \mathrm{m}), 7.81\left(4 \mathrm{H}, \mathrm{d},{ }^{3} J=7.7 \mathrm{~Hz}\right), 8.07$ $\left(4 \mathrm{H}, \mathrm{d},{ }^{3} \mathrm{~J}=8.5 \mathrm{~Hz}\right.$ ) (four $\mathrm{NH}$ protons were not assigned). ${ }^{13} \mathrm{C}$ NMR $\left(\mathrm{CDCl}_{3}, 298 \mathrm{~K}\right) \delta_{c} \mathrm{ppm}: 28.8(4 \mathrm{C}), 42.6(4 \mathrm{C}), 45.9(8 \mathrm{C}), 52.2(8 \mathrm{C})$, 58.5 (4C), 70.4 (4C), 70.6 (4C), 70.8 (4C), 103.3 (4C), 120.7 (4C), 122.3 (4C), 123.8 (4C), 124.1 (4C), 124.2 (4C), 125.8 (4C), 128.7 (4C), 133.1 (4C), 143.6 (4C).

Macrobicycle 9a. Obtained from compound 6 (318 mg, 0.5 mmol) and diamine $7 \mathbf{a}(37 \mathrm{mg}, 0.5 \mathrm{mmol})$. Eluent $\mathrm{CH}_{2} \mathrm{Cl}_{2} / \mathrm{MeOH} /$ $\mathrm{NH}_{3}$ aq 100:20:2. Yield $7 \mathrm{mg}(2.5 \%)$, beige crystalline powder, m.p. 192-194 ${ }^{\circ} \mathrm{C}$. (MALDI-TOF) found: 551.3820. $\mathrm{C}_{35} \mathrm{H}_{47} \mathrm{~N}_{6}$ requires $551.3862[\mathrm{M}+\mathrm{H}]^{+}$. UV $\left(\mathrm{CH}_{2} \mathrm{Cl}_{2}\right) \lambda_{\text {max }} \mathrm{nm}(\varepsilon): 336(6300) .{ }^{1} \mathrm{H} \mathrm{NMR}$ $\left(\mathrm{CDCl}_{3}, 298 \mathrm{~K}\right) \delta_{\mathrm{H}}$ ppm: $2.00(2 \mathrm{H}$, br.s $), 2.10\left(2 \mathrm{H}\right.$, quintet, ${ }^{3} J=5.9$ $\mathrm{Hz}), 2.29-2.81(18 \mathrm{H}, \mathrm{m}), 3.50$ (4H, br.s), $3.87(4 \mathrm{H}, \mathrm{s}), 4.87(2 \mathrm{H}$, br.s), $6.34\left(2 \mathrm{H}, \mathrm{d},{ }^{3} \mathrm{~J}=7.8 \mathrm{~Hz}\right), 6.81\left(2 \mathrm{H}, \mathrm{d},{ }^{3} \mathrm{~J}=7.8 \mathrm{~Hz}\right), 7.16-7.21$ $(2 \mathrm{H}, \mathrm{m}), 7.39-7.44(2 \mathrm{H}, \mathrm{m}), 7.60\left(2 \mathrm{H}, \mathrm{d},{ }^{3} J=8.4 \mathrm{~Hz}\right), 7.93(2 \mathrm{H}, \mathrm{d}$, ${ }^{3} \mathrm{~J}=8.7 \mathrm{~Hz}$ ) (two $\mathrm{NH}$ protons were not assigned). ${ }^{13} \mathrm{C} \mathrm{NMR}\left(\mathrm{CDCl}_{3}\right.$, $298 \mathrm{~K}) \delta_{\mathrm{c}} \mathrm{ppm}: 25.5$ (2C), 28.3 (1C), 43.0 (2C), 43.8 (2C), 49.1 (2C), $53.3(2 \mathrm{C}), 54.2(2 \mathrm{C}), 57.8(2 \mathrm{C}), 104.6(2 \mathrm{C}), 120.4(2 \mathrm{C}), 123.4$ (2C), 123.6 (2C), 123.7 (2C), 124.1 (2C), 125.4 (2C), 127.7 (2C), 133.9 (2C), 142.7 (2C).

Macrobicycle $\mathbf{9 b}$. Obtained from compound 6 (318 mg, 0.5 $\mathrm{mmol}$ ) and diamine $7 \mathbf{b}$ (44 mg, $0.5 \mathrm{mmol})$. Eluent $\mathrm{CH}_{2} \mathrm{Cl}_{2} / \mathrm{MeOH} /$ $\mathrm{NH}_{3}$ aq 100:20:3. Yield $11 \mathrm{mg}$ (4 \%), beige crystalline powder, m.p. 148-150 ${ }^{\circ} \mathrm{C}$. (MALDI-TOF) found: 565.3981. $\mathrm{C}_{36} \mathrm{H}_{49} \mathrm{~N}_{6}$ requires $565.4019[\mathrm{M}+\mathrm{H}]^{+}$. UV $\left(\mathrm{CH}_{2} \mathrm{Cl}_{2}\right) \lambda_{\max } \mathrm{nm}(\varepsilon): 340(8300) .{ }^{1} \mathrm{H}$ NMR $\left(\mathrm{CDCl}_{3}, 298 \mathrm{~K}\right) \delta_{\mathrm{H}}$ ppm: 1.74 (4H, br.s), 1.99 (4H, br.s), 2.40-2.75 (16H, m), 3.39 (4H, br.s), 3.98 (4H, br.s), 4.45 (2H, br.s), $6.11(2 \mathrm{H}$, d, $\left.{ }^{3} J=7.8 \mathrm{~Hz}\right), 6.33\left(2 \mathrm{H}, \mathrm{d},{ }^{3} J=7.8 \mathrm{~Hz}\right), 7.26-7.31(2 \mathrm{H}, \mathrm{m}),, 7.47-$ $7.52(2 \mathrm{H}, \mathrm{m}), 7.68\left(2 \mathrm{H}, \mathrm{d},{ }^{3} J=8.6 \mathrm{~Hz}\right), 8.18\left(2 \mathrm{H}, \mathrm{d},{ }^{3} J=8.2 \mathrm{~Hz}\right)$ (two $\mathrm{NH}$ protons were not assigned). ${ }^{13} \mathrm{C} \mathrm{NMR}\left(\mathrm{CDCl}_{3}, 298 \mathrm{~K}\right) \delta_{\mathrm{c}}$ ppm: 23.8 (2C), $26.3(2 \mathrm{C}), 41.8(2 \mathrm{C}), 43.9$ (2C), 48.5 (2C), 50.4 (2C), 55.4 (2C), 58.2 (2C), 104.2 (2C), 120.6 (2C), 122.9 (2C), 123.7 (2C), 124.1 (2C), 124.5 (2C), 125.6 (2C), 129.6 (2C), 133.2 (2C), 142.1 (2C).

Macrobicycle 9c. Obtained from compound 6 (318 mg, 0.5 mmol) and diamine $7 \mathbf{c}(86 \mathrm{mg}, 0.5 \mathrm{mmol})$. Eluent $\mathrm{CH}_{2} \mathrm{Cl}_{2} / \mathrm{MeOH} /$ $\mathrm{NH}_{3}$ aq 100:20:2. Yield $33 \mathrm{mg}$ (10\%), beige glassy compound. (MALDI-TOF) found: 649.4920. $\mathrm{C}_{42} \mathrm{H}_{61} \mathrm{~N}_{6}$ requires 649.4958 $[\mathrm{M}+\mathrm{H}]^{+}$. UV $\left(\mathrm{CH}_{2} \mathrm{Cl}_{2}\right) \lambda_{\max } \mathrm{nm}(\varepsilon): 340$ (12000). ${ }^{1} \mathrm{H} \mathrm{NMR}\left(\mathrm{CDCl}_{3}\right.$, $298 \mathrm{~K}) \delta_{\mathrm{H}}$ ppm: $1.16-1.38(12 \mathrm{H}, \mathrm{m}), 1.64\left(4 \mathrm{H}\right.$, quintet, $\left.{ }^{3} J=6.8 \mathrm{~Hz}\right)$, 1.77 (4H, br.s), 2.49-2.67 (16H, m), $3.20\left(4 \mathrm{H}, \mathrm{q},{ }^{3} J=5.0 \mathrm{~Hz}\right), 3.92$ $(4 \mathrm{H}, \mathrm{s}), 4.33(2 \mathrm{H}, \mathrm{br} . \mathrm{s}), 6.50\left(2 \mathrm{H}, \mathrm{d},{ }^{3} J=7.7 \mathrm{~Hz}\right), 7.29-7.36(6 \mathrm{H}$, $\mathrm{m})$, 7.74-7.77 $(2 \mathrm{H}, \mathrm{m}), 8.03-8.07(2 \mathrm{H}, \mathrm{m})$ (two $\mathrm{NH}$ protons were not assigned). ${ }^{13} \mathrm{C} \mathrm{NMR}\left(\mathrm{CDCl}_{3}, 298 \mathrm{~K}\right) \delta_{\mathrm{c}} \mathrm{ppm}: 25.9(2 \mathrm{C}), 26.3$ (2C), 28.0 (2C), 28.2 (2C), 29.4 (2C), 43.6 (2C), 44.2 (2C), 48.8 (2C), $53.0(2 \mathrm{C}), 53.4(2 \mathrm{C}), 56.0(2 \mathrm{C}), 103.8(2 \mathrm{C}), 120.3(2 \mathrm{C}), 122.4$ (2C), 123.6 (2C), 124.1 (2C), 124.2 (2C), 125.7 (2C), 128.6 (2C), $133.1(2 \mathrm{C}), 142.9$ (2C). 
Macrobicycle 9d. Obtained from compound 6 (214 mg, 0.34 mmol) and triamine $7 \mathbf{d}(45 \mathrm{mg}, 0.34 \mathrm{mmol})$. Eluent $\mathrm{CH}_{2} \mathrm{Cl}_{2} / \mathrm{MeOH}$ $3: 1$. Yield $15 \mathrm{mg}(7 \%)$, beige glassy compound. (MALDI-TOF) found: 608.4473. $\mathrm{C}_{38} \mathrm{H}_{54} \mathrm{~N}_{7}$ requires $608.4441[\mathrm{M}+\mathrm{H}]^{+} .{ }^{1} \mathrm{H} \mathrm{NMR}$ $\left(\mathrm{CDCl}_{3}, 298 \mathrm{~K}\right) \delta_{\mathrm{H}}$ ppm: 1.95-2.06 (8H, m), 2.40-2.75 (16H, m), $2.97\left(4 \mathrm{H}, \mathrm{t},{ }^{3} \mathrm{~J}=5.8 \mathrm{~Hz}\right), 3.31\left(4 \mathrm{H}, \mathrm{t},{ }^{3} J=6.3 \mathrm{~Hz}\right), 3.97(4 \mathrm{H}, \mathrm{s})$, $5.98\left(2 \mathrm{H}, \mathrm{d},{ }^{3} \mathrm{~J}=7.9 \mathrm{~Hz}\right), 6.85\left(2 \mathrm{H}, \mathrm{d},{ }^{3} J=7.9 \mathrm{~Hz}\right), 7.31-7.51(4 \mathrm{H}$, m), $7.85\left(2 \mathrm{H}, \mathrm{d},{ }^{3} J=8.5 \mathrm{~Hz}\right), 8.16\left(2 \mathrm{H}, \mathrm{d},{ }^{3} J=8.2 \mathrm{~Hz}\right)(\mathrm{NH}$ protons were not assigned).

Macrobicycle $\mathbf{9 h}$. Obtained from compound 6 (214 mg, 0.34 mmol) and dioxadiamine $7 \mathbf{h}(50 \mathrm{mg}, 0.34 \mathrm{mmol})$. Eluent $\mathrm{CH}_{2} \mathrm{Cl}_{2} /$ $\mathrm{MeOH} 3: 1$. Yield $10 \mathrm{mg}$ (5\%), yellow glassy compound. UV $\left(\mathrm{CH}_{2} \mathrm{Cl}_{2}\right) \lambda_{\max } \mathrm{nm}(\varepsilon): 339$ (11000). (MALDI-TOF) found: 625.4208 . $\mathrm{C}_{38} \mathrm{H}_{53} \mathrm{~N}_{6} \mathrm{O}_{2}$ requires $625.4230[\mathrm{M}+\mathrm{H}]^{+} .{ }^{1} \mathrm{H} \mathrm{NMR}\left(\mathrm{CDCl}_{3}, 298 \mathrm{~K}\right) \delta_{\mathrm{H}}$ ppm:1.87 (4H, br.s), 2.49 (4H, br.s), 2.65 (4H, t, $\left.{ }^{3} J=5.6 \mathrm{~Hz}\right), 2.69$ $\left(4 \mathrm{H}, \mathrm{t},{ }^{3} J=5.1 \mathrm{~Hz}\right), 2.75(4 \mathrm{H}, \mathrm{br} . \mathrm{s}), 3.32\left(4 \mathrm{H}, \mathrm{t},{ }^{3} J=5.7 \mathrm{~Hz}\right), 3.73$ $(4 \mathrm{H}, \mathrm{s}), 3.81\left(4 \mathrm{H}, \mathrm{t},{ }^{3} J=5.7 \mathrm{~Hz}\right), 3.83(4 \mathrm{H}, \mathrm{br} . \mathrm{s}), 6.09\left(2 \mathrm{H}, \mathrm{d},{ }^{3} J=\right.$ $7.8 \mathrm{~Hz}), 6.97\left(2 \mathrm{H}, \mathrm{d},{ }^{3} J=7.8 \mathrm{~Hz}\right), 7.36-7.41(2 \mathrm{H}, \mathrm{m}), 7.44-7.49$ $(2 \mathrm{H}, \mathrm{m}), 7.81\left(2 \mathrm{H}, \mathrm{d},{ }^{3} J=8.3 \mathrm{~Hz}\right), 8.02\left(2 \mathrm{H}, \mathrm{d},{ }^{3} J=8.5 \mathrm{~Hz}\right)(\mathrm{NH}$ protons were not assigned). ${ }^{13} \mathrm{C} \mathrm{NMR}\left(\mathrm{CDCl}_{3}, 298 \mathrm{~K}\right) \delta_{\mathrm{c}}$ ppm: 24.9 (2C), 43.8 (2C), 48.5 (2C), 49.7 (2C), 52.1 (2C), 54.3 (2C), 57.9 (2C), 69.1 (2C), 70.4 (2C), 104.5 (2C), 121.0 (2C), 122.7 (2C), 123.3 (2C), 123.8 (2C), 124.7 (2C), 126.0 (2C), 128.3 (2C), 132.7 (2C), 143.2 (2C).

Macrobicycle 9i. Obtained from compound 6 (214 mg, 0.34 $\mathrm{mmol}$ ) and dioxadiamine $7 \mathbf{i}(69 \mathrm{mg}, 0.34 \mathrm{mmol})$. Eluent $\mathrm{CH}_{2} \mathrm{Cl}_{2} /$ $\mathrm{MeOH} / \mathrm{NH}_{3}$ aq 100:20:2. Yield $23 \mathrm{mg}$ (10\%), yellow glassy compound. UV $\left(\mathrm{CH}_{2} \mathrm{Cl}_{2}\right) \lambda_{\max } \mathrm{nm}(\varepsilon): 340$ (11000). (MALDI-TOF) found: 681.4881. $\mathrm{C}_{42} \mathrm{H}_{61} \mathrm{~N}_{6} \mathrm{O}_{2}$ requires $681.4856[\mathrm{M}+\mathrm{H}]^{+}$. ${ }^{1} \mathrm{H} \mathrm{NMR}$ $\left(\mathrm{CDCl}_{3}, 298 \mathrm{~K}\right) \delta_{\mathrm{H}}$ ppm: 1.74 (4H, br.s), 1.83 (4H, br.s), $1.96(4 \mathrm{H}$, br.s), 2.45-2.50 (4H, m), 2.57-2.62 (4H, m), 2.65-2.72 (8H, m), 3.11 (4H, br.s), 3.48 (4H, br.s), $3.58\left(4 \mathrm{H}, \mathrm{t},{ }^{3} J=5.0 \mathrm{~Hz}\right), 3.81(4 \mathrm{H}, \mathrm{s})$, $5.22\left(2 \mathrm{H}\right.$, br.s), $6.21\left(2 \mathrm{H}, \mathrm{d},{ }^{3} J=7.8 \mathrm{~Hz}\right), 7.13\left(2 \mathrm{H}, \mathrm{d},{ }^{3} J=7.4 \mathrm{~Hz}\right)$, 7.16-7.21 (2H, m), 7.23-7.28 (2H, m), $7.73\left(2 \mathrm{H}, \mathrm{d},{ }^{3} J=8.3 \mathrm{~Hz}\right), 7.87$ $\left(2 \mathrm{H}, \mathrm{d},{ }^{3} \mathrm{~J}=8.5 \mathrm{~Hz}\right.$ ) (two $\mathrm{NH}$ protons were not assigned). ${ }^{13} \mathrm{C}$ NMR $\left(\mathrm{CDCl}_{3}, 298 \mathrm{~K}\right) \delta_{\mathrm{c}}$ ppm: $25.1(2 \mathrm{C}), 27.0$ (2C), 28.7 (2C), $43.4(2 \mathrm{C})$, $48.3(2 \mathrm{C}), 49.6(2 \mathrm{C}), 52.2(2 \mathrm{C}), 54.4(2 \mathrm{C}), 57.9(2 \mathrm{C}), 71.1$ (2C), 71.4 (2C), $102.6(2 \mathrm{C}), 120.9$ (2C), 121.9 (2C), 123.5 (2C), 123.7 (2C), 123.9 (2C), 125.6 (2C), 128.3 (2C), 132.5 (2C), 143.5 (2C).

Macrobicycle 9j. Obtained from compound 6 (318 mg, 0.5 mmol) and trioxadiamine $7 \mathbf{i}(110 \mathrm{mg}, 0.5 \mathrm{mmol})$. Eluent $\mathrm{CH}_{2} \mathrm{Cl}_{2} /$ $\mathrm{MeOH} / \mathrm{NH}_{3}$ aq 100:20:3. Yield $45 \mathrm{mg}$ (13\%), yellow glassy compound. UV $\left(\mathrm{CH}_{2} \mathrm{Cl}_{2}\right) \lambda_{\text {max }} \mathrm{nm}(\varepsilon): 340$ (11000). (MALDI-TOF) found: 697.4841. $\mathrm{C}_{42} \mathrm{H}_{61} \mathrm{~N}_{6} \mathrm{O}_{3}$ requires $697.4805[\mathrm{M}+\mathrm{H}]^{+}$. ${ }^{1} \mathrm{H}$ NMR $\left(\mathrm{CDCl}_{3}, 298 \mathrm{~K}\right) \delta_{\mathrm{H}}$ ppm: $1.82\left(4 \mathrm{H}\right.$, quintet, $\left.{ }^{3} \mathrm{~J}=5.8 \mathrm{~Hz}\right), 1.98(4 \mathrm{H}$, br.s), 2.50-2.60 (4H, m), 2.65-2.75 (4H, m), 2.90 (8H, br.s), 3.48-
$3.53(12 \mathrm{H}, \mathrm{m}), 3.60-3.64$ (4H, m), 3.85 (4H, s), 4.89 (2H, br.s), 6.01 $\left(2 \mathrm{H}, \mathrm{d},{ }^{3} J=7.7 \mathrm{~Hz}\right), 7.12\left(2 \mathrm{H}, \mathrm{d},{ }^{3} J=7.8 \mathrm{~Hz}\right), 7.26-7.31(4 \mathrm{H}, \mathrm{m})$, 7.72-7.75 $(2 \mathrm{H}, \mathrm{m}), 7.93-7.96(2 \mathrm{H}, \mathrm{m})$ (two $\mathrm{NH}$ protons were not assigned). ${ }^{13} \mathrm{C} \mathrm{NMR}\left(\mathrm{CDCl}_{3}, 298 \mathrm{~K}\right) \delta_{\mathrm{c}} \mathrm{ppm}: 26.0(2 \mathrm{C}), 28.5(2 \mathrm{C})$, 43.0 (2C), 48.4 (2C), 49.9 (2C), 54.0 (2C), 55.0 (2C), 56.3 (2C), 70.3 (2C), 70.4 (2C), $71.3(2 \mathrm{C}), 103.0$ (2C), 120.9 (2C), $121.7(2 \mathrm{C})$, 123.5 (2C), 123.7 (2C), 124.1 (2C), 125.3 (2C), 127.5 (2C), 132.7 (2C), 142.9 (2C).

\section{Results and Discussion}

In our previous investigations we successfully synthesized macrobicycles based on cyclen and cyclam central moieties containing benzyl ${ }^{[24-26]}$ and pyridinylmethyl spacers. ${ }^{[27]}$ The yields of the corresponding cryptands were shown to be dependent on the nature of the tetraazamacrocyclic fragment and the spacer used, the better results being obtained for cyclen derivatives, especially those with benzyl spacers. We have also shown the possibility to synthesize macrobicycles based on cyclam possessing two naphthylmethyl substituents on the nitrogen atoms of the cryptand. ${ }^{[28]}$ In this communication we report the synthesis of macrobicyclic derivatives of cyclen and cyclam with naphthylmethyl spacers which can be viewed as a valuable and simple chromophore and fluorophore group useful for the creation of macrocyclic chemosensors for metal cations.

For this purpose we synthesized 1-bromo-4(bromomethyl)naphthalene in $90 \%$ yield from commercially available 1-bromo-4-methylnaphthalene by its bromination with $\mathrm{NBS}$ in $\mathrm{CCl}_{4}$. Protected tetraazamacrocycles, cis-glyoxal-cyclen $\mathbf{1}$ and bis-formaldehyde-cyclam 2, were dialkylated in $\mathrm{MeCN}$ using this bromide to give disalts $\mathbf{3}$ and $\mathbf{4}$ in 88 and $76 \%$ yields respectively, and after a standard deprotection step (heating with $\mathrm{NaOH}$ in water) trans-bis(4-bromonaphth-1-ylmethyl) substituted cyclen and cyclam 5 and 6 were obtained in overall 87 and $46 \%$ yields (Scheme 1).

Compounds 5 and $\mathbf{6}$ were introduced in the Pd-catalyzed amination reactions with a series of di- and polyamines 7a-j (Scheme 2). Starting compounds were taken in equimolar<smiles>C1CN2CCN3CCN4CCN1C243</smiles><smiles>BrCc1ccc(Br)c2ccccc12</smiles>

2 equiv. $\mathrm{CH}_{3} \mathrm{CN}$<smiles>C1CCN(CCCN2CCN3CCCCN(CC3)C2)CC1</smiles>

2

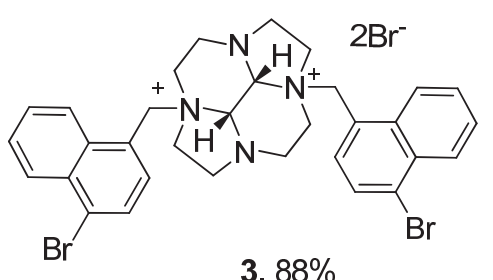

$\mathrm{NaOH}$ or $\mathrm{KOH} / \mathrm{H}_{2} \mathrm{O}$

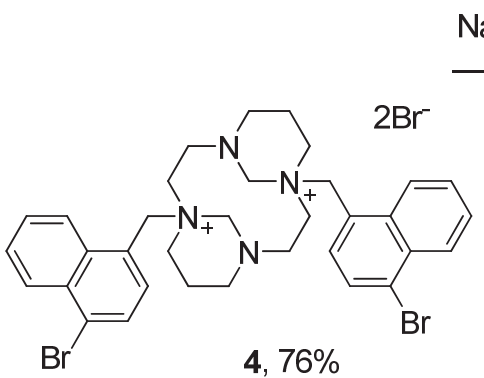

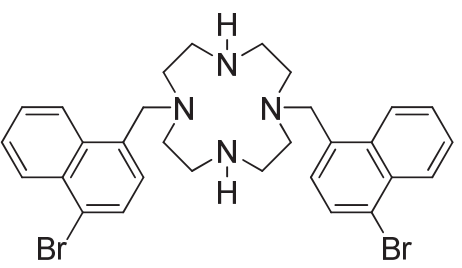

5, $87 \%$ (overall)

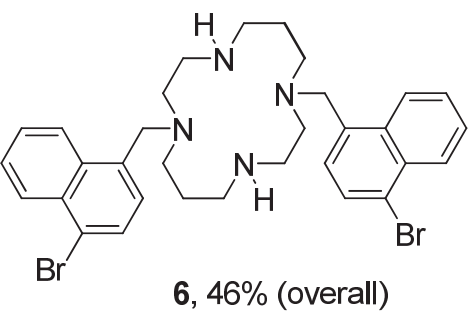

Scheme 1. 
amounts, the macrocyclization was catalyzed with $\operatorname{Pd}(\mathrm{dba})_{2} /$ DavePhos catalytic system $($ DavePhos $=2$-(dimethylamino)2'-(dicyclohexylphosphino)biphenyl) because initial experiments unexpectedly demonstrated low efficiency of a standard BINAP (2,2'-bis(diphenylphosphino)-1,1'binaphthalene) ligand. It was surprising because 1- and 2-bromonaphthalenes are known to be very active substrates in Pd-catalyzed amination reactions. ${ }^{[29-31]}$ This fact may be explained by the presence of the tetraazamacrocyclic fragment which competes with the phosphine ligand in the coordination of $\operatorname{Pd}(0)$ partially removing it from the catalytic cycle.

Target compounds $\mathbf{8}$ and $\mathbf{9}$ were obtained after column chromatography on silica gel, in order to isolate them from mixtures with macrotricyclic cyclodimers which were formed in comparable amounts. The use of the minimal amount of silica gel helped to obtain pure target cryptands. Cyclen-containing macrobicycles $\mathbf{8}$ were obtained in moderate yields, while the yields of cyclam-containing cryptands 9 were always low (Table 1). Higher yields of the cyclen derivatives compared to cyclam derivatives were observed by us earlier in the majority of cases where macrobicycles were synthesized. ${ }^{[27]}$ The cryptands yields do not notably depend on the chain length of polyamines but rather on the number of nitrogen atoms, it was noted that $\mathrm{NHCH}_{2} \mathrm{CH}_{2} \mathrm{NH}$ and $\mathrm{OCH}_{2} \mathrm{CH}_{2} \mathrm{O}$ fragments present in polyamine structure diminish the yields (Table 1, entries $5,6,8)$. It may be explained by a better coordination of $\operatorname{Pd}(0)$ by these fragments which form stable chelates and hinder amination reaction. It is interesting to note that in some cases the decrease in the catalyst loading from 16 to $8 \mathrm{~mol} \%$ together with the increase in the reagents concentration from 0.02 to $0.04 \mathrm{M}$ and application of 1.5 equiv. of polyamine led to better yields of the target macrobicycles 8 (entries 9, 12). The formation of cyclic dimers and higher mass oligomers was noted in all reactions, however, macrotricycles $\mathbf{1 0}$ were isolated only in the case of cyclen derivatives and in some cases their yields were even higher than those of target cryptands 8 (entries 4, 5, 11). Cyclic oligomers of higher masses have been never isolated as individual compounds, but in some cases corresponding signals were observed in MALDITOF spectra of mixtures.

\section{Conclusions}

As a result, we elaborated cryptands containing tetraazamacrocycle, two naphthylmethyl and polyamine moieties using Pd-catalyzed macrocyclization of easily avaialable trans- $N, N$ '-bis(naphthylmethyl) derivatives of cyclen and cyclam. The yields of the target macrobicycles were shown to be dependent on the nature of starting compounds, better yields being observed in the case of cyclen derivatives. Interesting macrotricyclic compounds were obtained as the second products in the reactions of the cyclen derivative with polyamines.

Acknowledgements. This work was carried out in the frame of the International Associated French-Russian Laboratory of Macrocycle Systems and Related Materials (LAMREM) of RAS and CNRS and financially supported by the RFBR grants 12-03-93107, 13-03-00813 and 13-03-90453. Generous provision of cyclen and cyclam derivatives by CheMatech Co is acknowledged.

Table 1. Synthesis of cryptands 8 and 9 (Pd(dba)/DavePhos, $16 / 16 \mathrm{~mol} \%, C=0.02 \mathrm{M})$.

\begin{tabular}{|c|c|c|c|c|}
\hline Entry & $\begin{array}{c}\text { Tetraazamacrocycle } \\
\text { derivative }\end{array}$ & Polyamine 7 & $\begin{array}{c}\text { Yields of } \\
\text { macrobicycles }\end{array}$ & $\begin{array}{l}\text { Yields of } \\
\text { cyclodimers }\end{array}$ \\
\hline 1 & 5 & $\mathrm{NH}_{2}\left(\mathrm{CH}_{2}\right)_{3} \mathrm{NH}_{2} 7 \mathbf{a}$ & $\mathbf{8 a}, 20 \%$ & $\mathbf{1 0 a}, 17 \%$ \\
\hline 2 & 5 & $\mathrm{NH}_{2}\left(\mathrm{CH}_{2}\right)_{4} \mathrm{NH}_{2} 7 \mathbf{b}$ & $\mathbf{8 b}, 7 \%$ & \\
\hline 3 & 5 & $\mathrm{NH}_{2}\left(\mathrm{CH}_{2}\right)_{10} \mathrm{NH}_{2} 7 \mathrm{c}$ & $\mathbf{8 c}, 17 \%$ & \\
\hline 4 & 5 & $\mathrm{NH}_{2}\left(\mathrm{CH}_{2}\right)_{3} \mathrm{NH}\left(\mathrm{CH}_{2}\right)_{3} \mathrm{NH}_{2} \mathbf{7 d}$ & $\mathbf{8 d}, 18 \%$ & 10d, $19 \%$ \\
\hline 5 & 5 & $\mathrm{NH}_{2}\left(\mathrm{CH}_{2}\right)_{2} \mathrm{NH}\left(\mathrm{CH}_{2}\right)_{3} \mathrm{NH}\left(\mathrm{CH}_{2}\right)_{2} \mathrm{NH}_{2} 7 \mathbf{e}$ & $\mathbf{8 e}, 9 \%$ & $10 \mathrm{e}, 12 \%$ \\
\hline 6 & 5 & $\mathrm{NH}_{2}\left(\mathrm{CH}_{2}\right)_{3} \mathrm{NH}\left(\mathrm{CH}_{2}\right)_{2} \mathrm{NH}\left(\mathrm{CH}_{2}\right)_{3} \mathrm{NH}_{2} 7 \mathbf{f}$ & $\mathbf{8 f}, 13 \%$ & $10 f, 6 \%$ \\
\hline 7 & 5 & $\mathrm{NH}_{2}\left(\mathrm{CH}_{2}\right)_{3} \mathrm{NH}\left(\mathrm{CH}_{2}\right)_{3} \mathrm{NH}\left(\mathrm{CH}_{2}\right)_{3} \mathrm{NH}_{2} 7 \mathbf{g}$ & $8 g, 17 \%$ & $\log , 4 \%$ \\
\hline 8 & 5 & $\mathrm{NH}_{2}\left(\mathrm{CH}_{2}\right)_{2} \mathrm{O}\left(\mathrm{CH}_{2}\right)_{2} \mathrm{O}\left(\mathrm{CH}_{2}\right)_{2} \mathrm{NH}_{2} 7 \mathbf{h}$ & $\mathbf{8 h}, 11 \%$ & $10 \mathrm{~h}, 6 \%$ a) \\
\hline 9 & 5 & $\mathrm{NH}_{2}\left(\mathrm{CH}_{2}\right)_{2} \mathrm{O}\left(\mathrm{CH}_{2}\right)_{2} \mathrm{O}\left(\mathrm{CH}_{2}\right)_{2} \mathrm{NH}_{2} 7 \mathbf{h}^{\mathrm{b})}$ & $\mathbf{8 h}, 29 \%$ & \\
\hline 10 & 5 & $\mathrm{NH}_{2}\left(\mathrm{CH}_{2}\right)_{3} \mathrm{O}\left(\mathrm{CH}_{2}\right)_{4} \mathrm{O}\left(\mathrm{CH}_{2}\right)_{3} \mathrm{NH}_{2} 7 \mathbf{i}$ & $\mathbf{8 i}, 13 \%$ & $\mathbf{1 0 i}, 8 \%$ \\
\hline 11 & 5 & $\mathrm{NH}_{2}\left(\mathrm{CH}_{2}\right)_{3}\left[\mathrm{O}\left(\mathrm{CH}_{2}\right)_{2}\right]_{2} \mathrm{O}\left(\mathrm{CH}_{2}\right)_{3} \mathrm{NH}_{2} 7 \mathbf{j}$ & $8 \mathbf{j}, 10 \%$ & $\mathbf{1 0 j}, 13 \%$ \\
\hline 12 & 5 & $\mathrm{NH}_{2}\left(\mathrm{CH}_{2}\right)_{3}\left[\mathrm{O}\left(\mathrm{CH}_{2}\right)_{2}\right]_{2} \mathrm{O}\left(\mathrm{CH}_{2}\right)_{3} \mathrm{NH}_{2} 7 \mathbf{j}^{\mathrm{b})}$ & $\mathbf{8 j}, 28 \%$ & \\
\hline 13 & 6 & $\mathrm{NH}_{2}\left(\mathrm{CH}_{2}\right)_{3} \mathrm{NH}_{2} 7 \mathbf{a}$ & 9a, $2.5 \%$ & \\
\hline 14 & 6 & $\mathrm{NH}_{2}\left(\mathrm{CH}_{2}\right)_{4} \mathrm{NH}_{2} 7 \mathbf{b}$ & $9 b, 4 \%$ & \\
\hline 15 & 6 & $\mathrm{NH}_{2}\left(\mathrm{CH}_{2}\right)_{10} \mathrm{NH}_{2} 7 \mathbf{c}$ & $9 c, 10 \%$ & \\
\hline 16 & 6 & $\mathrm{NH}_{2}\left(\mathrm{CH}_{2}\right)_{3} \mathrm{NH}\left(\mathrm{CH}_{2}\right)_{3} \mathrm{NH}_{2} \mathbf{7 d}$ & $9 d, 7 \%$ & \\
\hline 17 & 6 & $\mathrm{NH}_{2}\left(\mathrm{CH}_{2}\right)_{2} \mathrm{O}\left(\mathrm{CH}_{2}\right)_{2} \mathrm{O}\left(\mathrm{CH}_{2}\right)_{2} \mathrm{NH}_{2} 7 \mathbf{h}$ & $9 h, 5 \%$ & \\
\hline 18 & 6 & $\mathrm{NH}_{2}\left(\mathrm{CH}_{2}\right)_{3} \mathrm{O}\left(\mathrm{CH}_{2}\right)_{4} \mathrm{O}\left(\mathrm{CH}_{2}\right)_{3} \mathrm{NH}_{2} 7 \mathbf{i}$ & $9 \mathrm{i}, 10 \%$ & \\
\hline 19 & 6 & $\mathrm{NH}_{2}\left(\mathrm{CH}_{2}\right)_{3}\left[\mathrm{O}\left(\mathrm{CH}_{2}\right)_{2}\right]_{2} \mathrm{O}\left(\mathrm{CH}_{2}\right)_{3} \mathrm{NH}_{2} 7 \mathbf{j}$ & $\mathbf{9 j}, 13 \%$ & \\
\hline
\end{tabular}

a) Cyclodimer $\mathbf{1 0 h}$ was obtained in a mixture with macrobicycle $\mathbf{8 h}$.

b) The reaction was run using $8 / 9 \mathrm{~mol} \% \mathrm{Pd}(\mathrm{dba})_{2} /$ DavePhos, $C=0.04 \mathrm{M}$, and polyamine:5 mole ratio 1.5:1. 

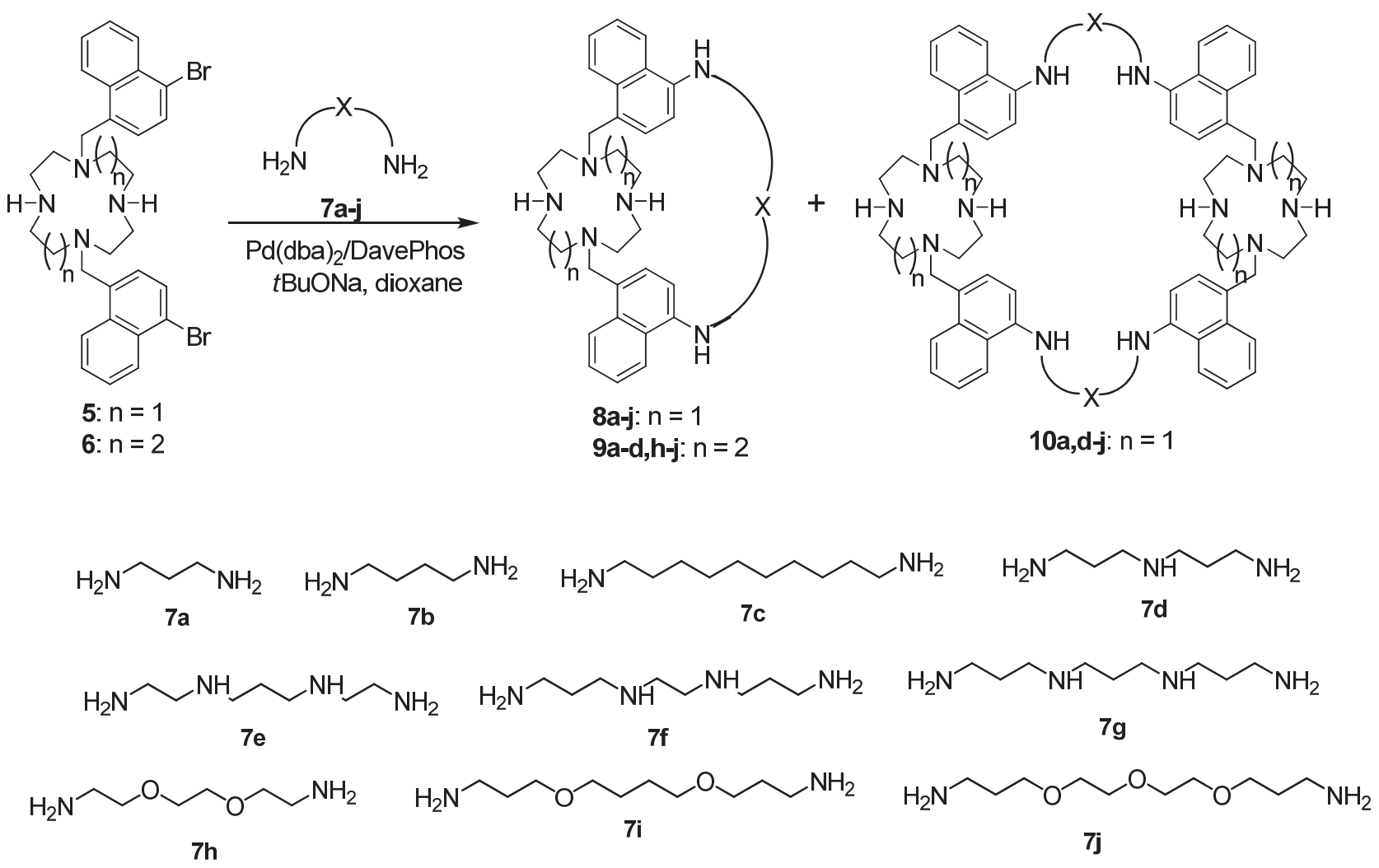

Scheme 2.

\section{References}

1. Lüttringhaus A. Liebigs Ann. Chem. 1937, 528, 181-210.

2. Yamashita T., Nakamura H., Takagi M., Ueno K. Bull. Chem. Soc. Jpn. 1980, 53, 1550-1554.

3. Koeni K.E., Lein G.M., Stuckler P., Kaneda T., Cram D.J. J. Am. Chem. Soc. 1979, 101, 3553-3566.

4. Helgeson R.C., Tarnowski T.L., Timko J.M., Cram D.J. J. Am. Chem. Soc. 1977, 99, 6411-6418.

5. Johnson M.R., Colburn C.A., Ganion S.J., Son B., Mosbo J.A., Sousa L.R. Magn. Reson. Chem. 1988, 26, 197-203.

6. Helgeson R.C., Weisman G.R., Toner J.L., Tarnowski T.L., Chao Y., Mayer J.M., Cram D.J. J. Am. Chem. Soc. 1979, 101, 4928-4941.

7. Helgeson R.C., Tarnowski T.L., Cram D.J. J. Org. Chem. 1979, 44, 2538-2550.

8. Colquhoun H.M., Goodings E.P., Maud J.M., Stoddart J.F., Williams D.J., Wolstenholme J.B. J. Chem. Soc., Chem. Commun. 1983, 1140-1142.

9. Colquhoun H.M., Goodings E.P., Maud J.M., Stoddart J.F., Wolstenholme J.B., Williams D.J. J. Chem. Soc., Perkin Trans. 2 1985, 607-624.

10. Artz S.P., DeGrandpre M.P., Cram D.J. J. Org. Chem. 1985, 50, 1486-1496.

11. Lehn J.-M., Simon J., Moradpour A. Helv. Chim. Acta 1978, 61, 2407-2418.

12. Poh B.L., Seah L.H., Lim C.S. Tetrahedron 1990, 46, 4379-4386.

13. Gallant A.J., Yun M., Sauer M., Yeung C.S., MacLachlan M.J. Org. Lett. 2005, 7, 4827-4830.

14. Sharghi H. Zare A. Synthesis 2006, 999-1004.

15. Khoshbin M.S., Ovchinnikov M.V., Khalid S., Mirkin C.A., Stern C., Zakharov L.N., Rheingold A.L. Chem. Asian J. 2006, 1, 686-692.

16. Eshghi H., Mirzaei M., Mehdi E., Shahry H. J. Chem. Res. 2007, 2007, 272-274.
17. Patra G.K., Datta D. Ind. J. Chem., Sect. A 2000, 39, 480-483.

18. Tran H.-A., Ashram M., Mizyed S., Thompson D.W., Georghiou P.E. J. Inclusion Phenom. Macrocyclic Chem. 2008, 60, 43-49.

19. Lukyanenko N.G., Lyapunov A.Yu., Kirichenko T.I., Botoshansky M.M., Simonov Yu.A., Fonari M.S. Tetrahedron Lett. 2005, 46, 2109-2112

20. Kieran A.L., Pascu S.I., Jarosson T., Maxwell J., Sanders J.K.M. Chem. Commun. 2005, 1842-1844.

21. Qin H., He Y., Qing G., Hu C., Yang X. Tetrahedron: Assymetry 2006, 17, 2143-2148.

22. Alfonso I., Burguete M.I., Galindo F., Luis S.V., Vigara L. J. Org. Chem. 2007, 72, 7947-7956.

23. Ukai T., Kawazura H., Ishii Y., Bonnet J.J., Ibers J.A. J. Organomet. Chem. 1974, 65, 253-266.

24. Averin A.D., Shukhaev A.V., Buryak A.K., Denat F., Guilard R., Beletskaya I.P. Tetrahedron Lett. 2008, 49, 3950-3954.

25. Kobelev S.M., Averin A.D., Buryak A.K., Denat F., Guilard R., Beletskaya I.P. Heterocycles 2011, 82, 1447-1476.

26. Kobelev S.M., Averin A.D., Buryak A.K., Savelyev E.N., Orlinson B.S., Butov G.M., Novakov I.A., Denat F., Guilard R., Beletskaya I.P. ARKIVOC 2012, vii, 196-209.

27. Averin A.D., Tyutenov K.S., Shukhaev A.V., Kobelev S.M., Buryak A.K., Denat F., Guilard R., Beletskaya I.P. Heterocycles 2012, 86, 1341-1366.

28. Kobelev S.M., Averin A.D., Maloshitskaya O.A., Denat F., Guilard R., Beletskaya I.P. Macroheterocycles 2012, 5, 389-395.

29. Beletskaya I.P., Bessmertnykh A.G., Averin A.D., Denat F., Guilard R. Eur. J. Org. Chem. 2005, 261-280.

30. Averin A.D., Uglov A.N., Beletskaya I.P. Chem. Lett. 2008, 37, 1074-1075.

31. Uglov A.N., Averin A.D., Buryak A.K., BessmertnykhLemeune A., Guilard R., Beletskaya I.P. Macroheterocycles 2013, 6, 33-39. 